\title{
REVIEW
}

\section{Advances in InGaAs/InP single-photon detector systems for quantum communication}

\author{
Jun Zhang ${ }^{1,2}$, Mark A Itzler ${ }^{3}$, Hugo Zbinden ${ }^{4}$ and Jian-Wei Pan ${ }^{1,2}$
}

Single-photon detectors (SPDs) are the most sensitive instruments for light detection. In the near-infrared range, SPDs based on III-V compound semiconductor avalanche photodiodes have been extensively used during the past two decades for diverse applications due to their advantages in practicality including small size, low cost and easy operation. In the past decade, the rapid developments and increasing demands in quantum information science have served as key drivers to improve the device performance of single-photon avalanche diodes and to invent new avalanche quenching techniques. This Review aims to introduce the technology advances of InGaAs/InP single-photon detector systems in the telecom wavelengths and the relevant quantum communication applications, and particularly to highlight recent emerging techniques such as high-frequency gating at $\mathrm{GHz}$ rates and free-running operation using negative-feedback avalanche diodes. Future perspectives of both the devices and quenching techniques are summarized.

Light: Science \& Applications (2015) 4, e286; doi:10.1038/lsa.2015.59; published online 8 May 2015

Keywords: avalanche photodiode; detector; InGaAs/InP; quantum communication; single-photon avalanche diode; single-photon detection

\section{INTRODUCTION}

A single photon is the indivisible minimum energy unit of light, and therefore, detectors with the capability of single-photon detection are ultimate tools for weak light detection. ${ }^{1,2}$ So far, single-photon detectors (SPDs) have been widely used in numerous applications such as quantum communication, quantum information processing, Lidar and photoluminescence. Most near-infrared SPDs can be sorted into three principal categories of devices: photomultiplier tubes, superconducting devices and semiconductor single-photon avalanche diodes (SPADs). Apart from these devices, there are also some new technologies for single-photon detection such as quantum-dot optically gated field-effect transistor ${ }^{3}$ and quantum dot resonant tunneling diodes. ${ }^{4}$ Up-conversion detectors ${ }^{5-7}$ combining the nonlinear optical process of sum frequency generation and Si SPADs are still considered to belong to semiconductor devices.

Photomultiplier tubes are operated in high-vacuum tubes with high voltages between anodes and photocathodes. ${ }^{8}$ A primary electron is produced in the photocathode material as a consequence of the photoelectric effect, and high gain results from a multiplication mechanism that creates secondary electrons. Photomultiplier tubes can have large active areas, but they suffer from low efficiency and high dark count rate.

Superconducting SPDs include superconducting nanowire singlephoton detectors (SNSPD), ${ }^{9}$ transition edge sensors ${ }^{10}$ and superconducting tunnel junctions. ${ }^{11}$ In the SNSPD, a hotspot is created after the absorption of a single photon in superconducting nanowires, and subsequently, the superconducting current density increases due to the size expansion of the hotspot. Once the superconducting current density in the nanowires reaches the critical value, the nanowires are changed from the superconducting state to the normal resistance state. This transition generates a voltage signal of single-photon detection. The primary advantages of SNSPDs are low dark count rate, high photon count rate and very accurate time resolution. The detection efficiency was low (at the level of a few percent) for early generation devices, but recently, this parameter has been significantly improved through the efforts of the SNSPD community. ${ }^{12}$ However, the cryogenic operating conditions required for SNSPDs limit their use for practical applications.

Currently the mainstream solution for single-photon detection in practical applications is the use of SPADs. In the literature covering photodetectors, one finds the terminologies of avalanche photodiode (APD) and SPAD. Normally, a device is referred to as an APD when it operates below the breakdown voltage in the linear-mode, for which the output photocurrent is linearly proportional to the input optical power. The term SPAD refers to a device operated in Geiger mode, for which biasing above the breakdown voltage can result in a self-sustaining avalanche in response to the absorption of just a single photon. For a SPAD based detector system, there are two crucial parts: the SPAD device $^{13-15}$ and the quenching electronics. ${ }^{16-18}$ Therefore, the performance of SPD system depends both on the SPAD device itself and the quenching electronics as well.

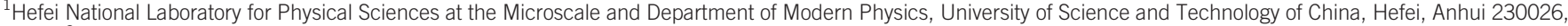

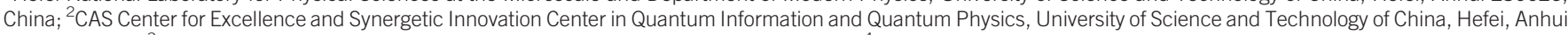

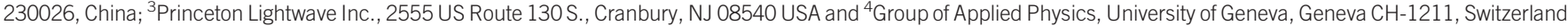

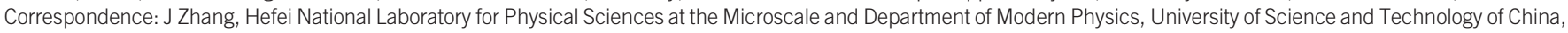
Hefei, Anhui 230026, China

E-mail: zhangjun@ustc.edu.cn

Received 3 December 2014; revised 29 January 2015; accepted 1 February 2015; accepted article preview online 5 February 2015 
In the following sections, we will first introduce the basic semiconductor structure, device performance improvement, and Geiger mode operations for InGaAs/InP SPADs. The characteristics and the characterization methods relevant to InGaAs/InP SPADs are subsequently presented, and then we will focus on recent advances of quenching techniques, particularly in the regimes of low-frequency gating, high-frequency gating and free-running operation. The applications for diverse quantum communication protocols such as quantum key distribution (QKD) ${ }^{19,20}$ quantum teleportation, ${ }^{21}$ quantum secret sharing (QSS), ${ }^{22-24}$ quantum secure direct communication $^{25-28}$ and counterfactual quantum cryptography $^{29}$ using InGaAs/InP SPADs will also be described briefly and representatively, and finally we conclude with a discussion of future perspectives on both SPAD devices and quenching techniques.

Relevant reviews concerning quantum cryptography, ${ }^{30,31}$ quantum communication, ${ }^{32,33}$ SPDs for quantum information applications, ${ }^{1}$ single-photon sources and detectors, ${ }^{2}$ solid-state SPDs ${ }^{34}$ and avalanche photodiodes ${ }^{35,36}$ could also be of significance to the reader as references.

\section{INGAAS/INP SPAD}

For the single-photon detection in the near-infrared, group III-V heterostructure devices such as InGaAs/InP and InGaAs/InAlAs with separate absorption, grading, charge and multiplication structures ${ }^{35,36}$ as shown in Figure 1 are the primary candidates. In these devices, an InGaAs $\left(\mathrm{In}_{0.53} \mathrm{Ga}_{0.47} \mathrm{As}\right)$ layer with a room-temperature band gap $E_{\mathrm{g}}$ of $0.75 \mathrm{eV}$ and a cutoff wavelength of around $1670 \mathrm{~nm}$ is used as the absorption material, while the lattice-matched InP layer or InAlAs layer is used as the multiplication material (Figure 1). The electric field in the multiplication layer is sufficiently high to provide the desired avalanche probability, while the electric field in the absorption layer is adequately low to minimize field-induced leakage currents. ${ }^{13}$ The charge layer is designed to provide high electric field in the multiplication layer and low electric field in the absorption layer, while the grading layer avoids carrier accumulation in the heterojunction interface. ${ }^{13}$ To improve SPAD performance, both the device structure design and device fabrication should be optimized specifically for single-photon detection. ${ }^{37-39}$

In Geiger mode, the reverse bias voltage of the $\operatorname{SPAD}\left(V_{\mathrm{b}}\right)$ is larger than the breakdown voltage $\left(V_{\mathrm{br}}\right)$. When a photon is absorbed, an electron-hole pair of electrical carriers is created. One carrier is subsequently injected into the depletion zone of multiplication layer and may initiate a self-sustaining avalanche due to the impact ionization mechanism at high electric field (on the order of $10^{5} \mathrm{~V} \mathrm{~cm}^{-1}$ ). The avalanche current reaches a macroscopic steady state within a buildup time on the order of a few hundred picoseconds.

The device structure of the InGaAs/InP SPAD illustrated in Figure 1 bears similarities to that of more mature 'linear-mode' APDs used at modest gains below their breakdown voltage. However, despite these structural similarities, the optimization of SPAD performance is significantly different from that of linear-mode APDs because these two device types are employed in dramatically different contexts. ${ }^{13}$ Linearmode APDs can provide sensitivity improvements in optical receivers (relative to more conventional receivers based on $\mathrm{p}-\mathrm{i}-\mathrm{n}$ photodiodes, which lack gain) as long as the noise of the APD is less than the noise of the amplifier which follows the APD in the receiver circuit. In general, linear-mode APDs only provide a sensitivity advantage for highbandwidth (e.g., $>1 \mathrm{GHz}$ ) receivers in which the necessarily broad frequency response leads to high amplifier noise. Therefore, linear-mode APD design emphasizes low excess noise and high bandwidth. ${ }^{35,36}$

In contrast, the role of SPADs is to provide an avalanche response that is sufficiently large to reliably detect the injection of a single photo-excited carrier into the multiplication region (Figure 1). This behavior is achieved by operating in Geiger mode (i.e., above $V_{\mathrm{br}}$ ), and in this capacity, the SPAD is more appropriately described as a photon-activated switch with an essentially digital response that is noise-free, at least in the sense that the threshold for detecting avalanches can be set far beyond the level of any background circuit noise. The only noise in a SPAD originates in 'dark counts' induced by thermal or field-mediated mechanisms in the absence of input signal photons. While the average dark count level can be subtracted from the overall device output, the shot noise of these dark counts is unavoidable.

The different operating modes for linear-mode APDs and SPADs require optimization of distinct performance attributes. For instance, linear-mode operation benefits from a high gain-bandwidth product, and since gain-bandwidth product is nominally inversely proportional to the width of the multiplication region $\left(W_{\mathrm{m}}\right)$, linear-mode APD design tends to emphasize narrow $W_{\mathrm{m}}$ of well under $1 \mu \mathrm{m}$. Conversely, gain-bandwidth product bears no direct impact on

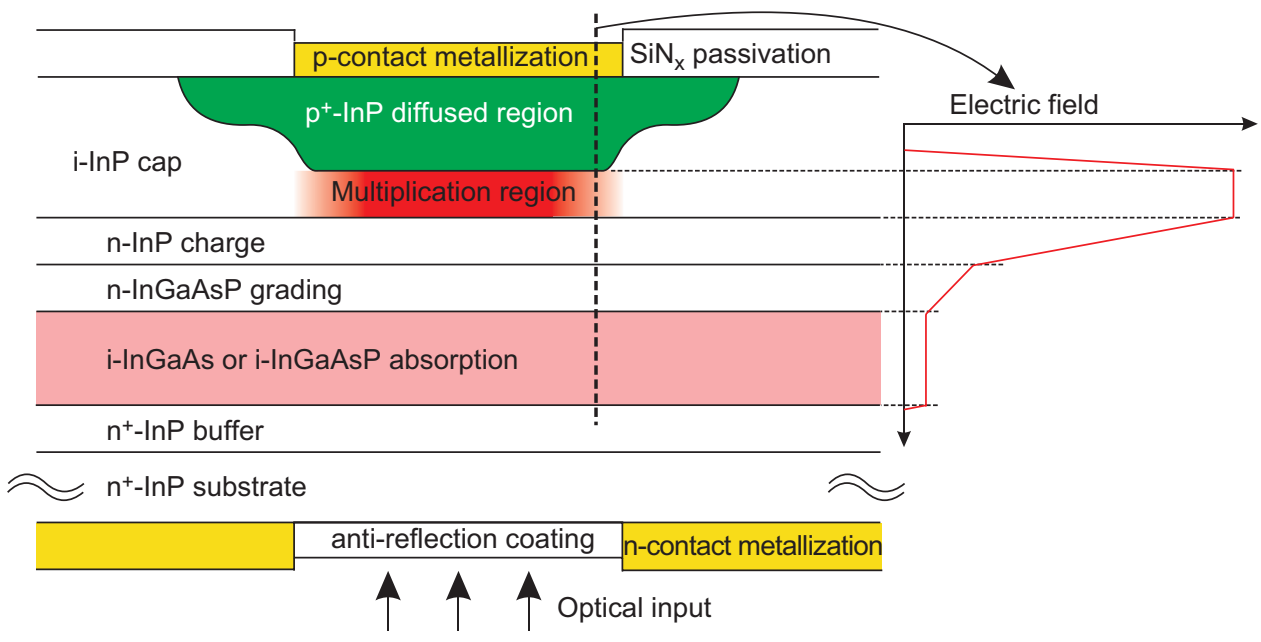

Figure 1 The SAGCM structure of InGaAs/InP SPAD. ${ }^{13}$ Figure reproduced: Ref. 13 @ 2007, Taylor \& Francis. SAGCM, separate absorption, grading, charge and multiplication; SPAD, single-photon avalanche diode. 
SPAD performance. Instead, wider multiplication regions can provide lower breakdown fields with a consequently smaller tunneling contribution to the dark counts, and so SPAD design tends to emphasize wider $W_{\mathrm{m}}$ of well beyond $1 \mu \mathrm{m} .{ }^{37,40}$ In a similar vein, linear-mode APDs benefit from lower excess noise, which can be provided by narrower multipliers, particularly when dead-space effects are prevalent. ${ }^{41}$ However, excess noise is not directly relevant to SPAD performance, and design considerations related to excess noise are absent during SPAD design.

From the perspective of underlying materials properties and fabrication technology in the InGaAsP material system, the sources of noise in linear-mode APDs and SPADs are also considerably different. One key consideration is that the dark current in state-of-the-art InGaAs/InP avalanche diode structures (including both linear-mode APDs and SPADs) is dominated by perimeter leakage mechanisms. ${ }^{42}$ Although this perimeter leakage does not pass through the multiplication region and remains unmultiplied, for InGaAs/InP avalanche diodes at typical operating temperatures, it is still dramatically larger than the multiplied bulk leakage current. Therefore, the dark current performance of linear-mode APDs is dominated by perimeter leakage, and improvements in this device type will require wafer fabrication improvements such as better surface passivation techniques. For SPADs, however, because the perimeter leakage is not multiplied, it does not induce detection events, and only the multiplication of bulk dark carriers influences the dark counts. Therefore, the most profitable strategy for improvement beyond the state-of-the-art dark count in SPADs will include a focus on the bulk material properties of the base epitaxial wafers.

Beyond these considerations of underlying SPAD device design, overall device performance is critically dependent on the detection circuitry that follows the SPAD. An appropriate circuit, referred to as quenching electronics, ${ }^{16}$ is necessary to rapidly suppress the avalanche by lowering the reverse bias down to $V_{\mathrm{br}}$, to output the detection signal of the incident photon by discriminating the leading edge of the avalanche current and to restore the SPAD to its armed state to detect the next incoming photon. Note that rapid quenching also reduces afterpulsing as we will discuss below, therefore the quenching electronics plays a key role in a SPAD system. Quenching technologies include passive quenching, active quenching, gated quenching and hybrid quenching. ${ }^{16}$

In a passive quenching circuit, ${ }^{43}$ a SPAD is connected with a reverse bias through a high-value ballast resistor (on the order of $100 \mathrm{k} \Omega$ ). When avalanches occur, the voltage difference between the anode and the cathode of the SPAD decreases swiftly due to the voltage drop across the resistor. Once the voltage difference is lowered sufficiently close to $V_{\mathrm{br}}$, avalanches will spontaneously quench. For a welldesigned SPAD and associated quenching circuit, the quenching time, defined as the time duration from avalanche occurrence to avalanche termination, is on the order of $1 \mathrm{~ns}$. Passive quenching is well suited for asynchronous single-photon detection (free-running mode). However, this technique suffers from long recovery times on the order of 100 ns due to the large time constant of resistance capacitance (RC).

To solve the problem of slow recovery in passive quenching, active quenching is implemented ${ }^{44,45}$ by using fast discrimination electronics to sense the leading edges of avalanches. The output signals of the discrimination electronics are used to switch the SPAD bias below $V_{\mathrm{br}}$, and the device is maintained in this disarmed state for a certain time period called the hold-off time or deadtime. Following the holdoff time, the bias is actively switched back to the initial armed state. In this scheme, both the quenching time and the recovery time can be a few nanoseconds or less using commercially available electronics components.

Gated quenching ${ }^{46}$ is widely used for applications requiring synchronous single-photon detection such as QKD. ${ }^{30}$ In such a scheme, gate pulses with a repetition frequency of $f_{\mathrm{g}}$, voltage amplitude of $V_{\mathrm{g}}$ and time duration of $\tau_{\mathrm{g}}$ (with a resulting duty cycle of $\tau_{\mathrm{g}} f_{\mathrm{g}}$ ) are coupled to a SPAD. The SPAD is working in Geiger mode only when the pulses are gated on. However, avalanche signals generated during the gate pulses are superposed with parasitic derivative signals that result from capacitive responses of the SPAD to the gate pulses. The key technical challenge in gated quenching is to extract avalanche signals from the background capacitive responses.

Each quenching technique has its own advantages and disadvantages. Therefore, hybrid quenching schemes combining the above techniques are sometimes applied. For instance, the scheme of passive quenching and active reset, which will be introduced in the later section, can efficiently shorten the recovery time. In the gated quenching scheme, for long gate width, the use of active quenching instead of gated quenching can significantly reduce the quenching time.

\section{CHARACTERISTICS AND CHARACTERIZATION OF SPAD}

For a SPAD-based SPD system, there are quite a few parameters that are important for performance evaluation, and optimization of one parameter often involves performance tradeoffs with other parameters. In this section, we first introduce the definitions and the mechanisms of these parameters, and then describe the experimental characterization.

The first important parameter is (single) photon detection efficiency (PDE), ${ }^{40}$ defined as the probability that the detector system produces a desired output signal in response to the arrival of an incident photon. From the view of SPAD structure, PDE is determined by $\mathrm{PDE}=\eta_{\text {coup }} \times \eta_{\text {abs }} \times \eta_{\text {inj }} \times \eta_{\text {ava }}$, where $\eta_{\text {coup }}$ is the coupling efficiency of SPAD, $\eta_{\text {abs }}$ is the absorption efficiency or (internal) quantum efficiency in the absorption layer of SPAD, $\eta_{\text {inj }}$ is the collection efficiency of the photo-excited carriers injected from the absorption layer to the multiplication layer and $\eta_{\text {ava }}$ is the probability of a detectable avalanche given the successful injection of a carrier into the multiplication layer. $\eta_{\text {coup }}$ depends on multiple factors such as insertion loss, surface reflectance and active area of device. $\eta_{\mathrm{abs}}$ is calculated by $\eta_{\text {abs }}=1-e^{-\alpha d}$, where $\alpha$ is the absorption coefficient and $d$ is the absorption depth. For InGaAs, $\alpha$ is around $7500 \mathrm{~cm}^{-1}$ at $1550 \mathrm{~nm}$ illumination. Given $d=1.5 \mu \mathrm{m}, \eta_{\text {abs }}$ is $0.68 . \eta_{\text {inj }}$ and $\eta_{\text {ava }}$ have a sensitive dependence on the electric field, which is determined by the excess bias $\left(V_{\mathrm{ex}}\right)$ defined as $V_{\mathrm{ex}}=V_{\mathrm{b}}-V_{\mathrm{br}}$.

Dark count rate (DCR) ${ }^{47}$ is used to characterize the noise performance of the detector system. DCR is defined as the normalized count rate in the absence of illumination. DCR depends on the conditions of temperature $(T)$ and $V_{\mathrm{ex}}$. Dark counts originate from the mechanisms of thermal excitation, tunneling excitation or trapassisted tunneling excitation. At sufficiently high operation temperatures, thermal excitation will be the dominant contribution to DCR, while at low temperatures or high electric fields (large $V_{\text {ex }}$ ), tunneling excitation will dominate the contributions to DCR. ${ }^{13}$ Although DCR is analogous to APD dark current in the linear-mode-e.g., the shot noise of both of these phenomenon plays a comparable role in their overall noise performance-DCR is generally not correlated with the device dark current measured in the linear-mode. This is due to the fact that dark current in the linear-mode is usually dominated by perimeter leakage currents that do not flow through the 
multiplication region and therefore do not result in Geiger mode avalanches.

Afterpulse probability $\left(P_{\mathrm{ap}}\right)$ is another important parameter of SPAD. During an avalanche, some carriers are trapped by defects and impurities in the multiplication layer. Subsequently these carriers are released and can initiate new undesired avalanches called afterpulses. ${ }^{48,49} P_{\text {ap }}$ is defined as the probability of producing afterpulsing counts due to the previous photon detection during a time period. Reducing $P_{\text {ap }}$ to an appropriately low level is crucial for most applications. Improving the crystal quality of multiplication material can effectively suppress the afterpulsing effect, but advances in fundamental material quality such as significant reduction in defect density are likely to take many years given the current relative maturity of the InGaAsP materials system. Alternatively, reducing the quantity of charge carriers during the avalanche process or shortening the lifetime of trapped carriers can also decrease $P_{\text {ap }}$. For a detector system, $P_{\text {ap }}$ is related to multiple conditions, which can be roughly modeled as $P_{\mathrm{ap}} \propto\left(C_{\mathrm{d}}+C_{\mathrm{p}}\right) \times \int_{0}^{\delta} V_{\mathrm{ex}}(t) \mathrm{d} t \times e^{-\tau_{\mathrm{d}} / \tau}$, where $C_{\mathrm{d}}$ is the diode capacitance, $C_{\mathrm{p}}$ is the parasitic capacitance of circuit including the lead capacitance of device, $\delta$ is the avalanche duration time, $\tau_{\mathrm{d}}$ is the hold-off time and $\tau$ is the lifetime of detrapping carriers. From the above equation, the approaches to reduce $P_{\text {ap }}$ include: (1) minimizing $C_{\mathrm{p}}$; (2) limiting $\delta$; (3) lowering $V_{\mathrm{ex}}$; (4) increasing $\tau_{\mathrm{d}}$; and (5) decreasing $\tau$ by increasing the operation temperature. However, these approaches also have consequent disadvantages. For instance, approach (1) and approach (2) can effectively reduce avalanche charge quantity, but the technical challenge is to extract weak avalanches from background noise. Approach (3) decreases PDE. Approach (4) limits the maximum count rate. Approach (5) increases DCR. Therefore, obtaining the desired afterpulsing performance can often force compromises with other SPAD performance parameters.

Timing jitter (time resolution) is usually defined as the total time uncertainty between incident photons and corresponding electrical signal outputs, which includes the contributions of the SPAD itself and the quenching electronics as well. The intrinsic timing jitter of the SPAD device strongly depends on $V_{\mathrm{ex}}$. With large $V_{\mathrm{ex}}$, high electric field greatly shortens avalanche build-up time and also its time uncertainty.

Maximum count rate is determined by the capability of the SPAD in the limit of saturated photon detections, which can be approximated by the reciprocal of $\tau_{\mathrm{d}}$. The ratio of the maximum count rate and the DCR determines the dynamic range of the SPAD.

Photon number resolution (PNR) is important for specific applications such as quantum computation and quantum optics. With conventional techniques of gating mode and free-running mode, the SPAD is believed not to have the capability of PNR since avalanches always reach the saturation stage due to relatively long quenching time. Operated in such a fashion, the SPAD can only resolve between zero photons and non-zero photons. Using a SPAD array (multipixels) or time-multiplexing scheme ${ }^{50}$ can implement PNR with conventional quenching techniques. Recently, experiments have shown that with ultra-short quenching time as described in later section, for instance, using the technique of high-speed gating, SPAD can also exhibit PNR capability.

The methods to characterize these parameters include the singlephoton scheme $e^{51,52}$ and photon pair scheme. ${ }^{53}$ In the single-photon scheme, ${ }^{51,52}$ a calibrated optical power meter with high accuracy and low uncertainty is used to measure the power of a pulsed laser, and a calibrated attenuator is used to highly attenuate the laser power down to the single-photon level. Therefore, the calibration for these optical instruments themselves is crucial in such a method. In the photon pair scheme ${ }^{53}$ correlated photon pairs are generated via a nonlinear optical technique such as spontaneous parametric down-conversion, in which signal and idler photons are sent to two detectors for calibrating and triggering, respectively. The output signals of detectors are further processed by a coincidence counter. In this method, the SPAD parameters, particularly PDE, can be precisely measured without requiring a calibrated power meter. Nevertheless, coupling efficiency in each channel is an important factor to be carefully considered.

A typical experimental set-up of the single-photon calibration method is shown in Figure 2. The SPAD is working in the gated mode with low repetition frequency $\left(f_{\mathrm{g}}\right)$, e.g., $10 \mathrm{kHz}$. A signal generator outputs original gates and synchronized signals with the same frequency to drive a laser diode. Short optical pulses emitted from the laser diode are divided by a beam splitter. One port of the beam splitter is monitored by a power meter and the other one is connected with a variable attenuator. The power of pulses after the attenuation reaches quasi-single-photon level. The detection signals produced in the quenching electronics are finally connected with a counter. To well illustrate the calibration and the tradeoffs of the SPAD parameters,

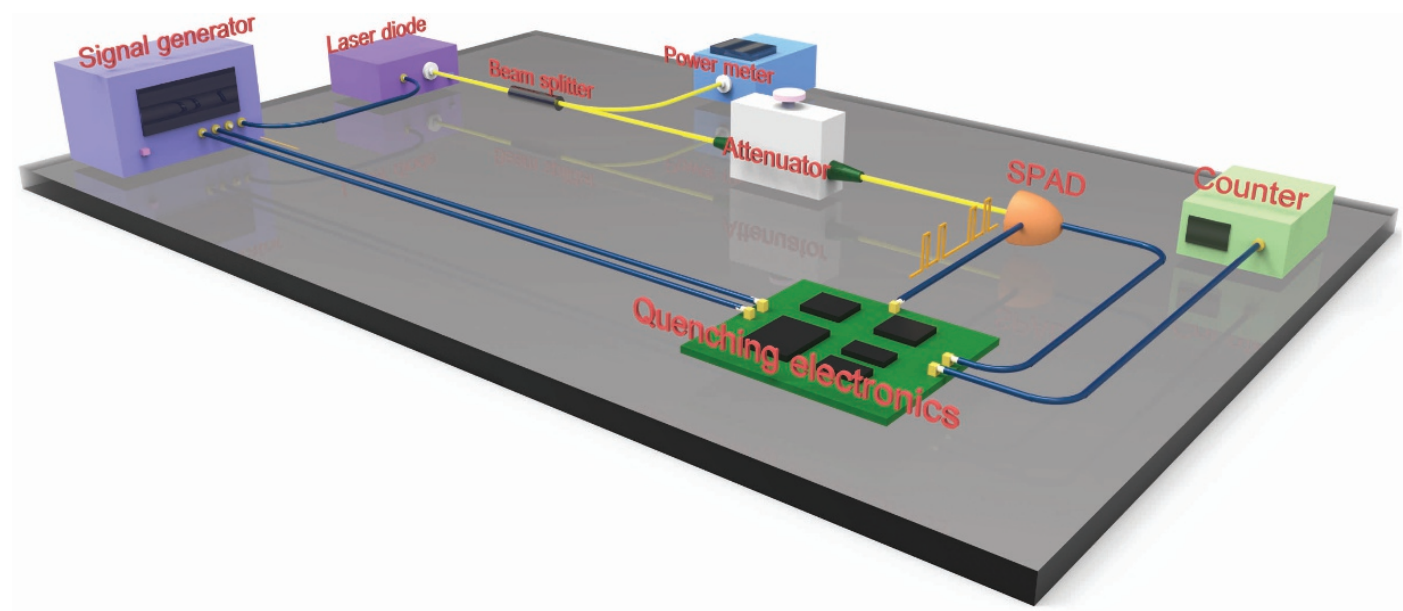

Figure 2 Single-photon test bench for SPAD performance calibration. SPAD, single-photon avalanche diode. 
we construct an experimental single-photon test bench according to Figure 2 and characterize an InGaAs/InP SPAD as an example. The typical experimental results are plot in Figure 3.

Given that measured count rates are $C_{\text {on }}$ and $C_{\text {off }}$ with and without laser illumination, respectively, the normalized DCR can be calculated as $\mathrm{DCR}=\frac{C_{\text {off }}}{f_{\mathrm{g}} t_{\mathrm{w}}} \times 10^{9}$, where $t_{\mathrm{w}}$ is the effective gating width (in ns). Considering the Poisson distribution of incident photons, $\mathrm{PDE}$ can be calculated as $\mathrm{PDE}=\frac{1}{\mu} \ln \frac{1-C_{\mathrm{off}} / f_{\mathrm{g}}}{1-C_{\mathrm{on}} / f_{\mathrm{g}}}$, where $\mu$ is the mean photon number per optical pulse.

The characterization of PDE and DCR as $V_{\text {ex }}$ linearly increases is shown in Figure 3a. Apparently, PDE is a linearly function and DCR is an exponential function of $V_{\mathrm{ex}}$. Therefore, the relationship between DCR and PDE is exponential.

To precisely characterize $P_{\text {ap }}$, double-gate method $^{51}$ is widely used. The first gate is for photon detection while the second gate with tunable delay relative to the first gate is for measuring afterpulse counts. In such a way, by varying the delay between the two gates the afterpulsing distribution can be clearly plotted after the subtraction of DCR contribution.

The experimental results of the afterpulsing distribution at different temperatures are plot in Figure $3 \mathrm{~b}$, in which the normalized afterpulse probabilities $\left(P_{\mathrm{ap}} / t_{\mathrm{w}}\right)$ exponentially decay in time. The gap between the two lines in Figure $3 \mathrm{~b}$ clearly shows that increasing temperature can effectively suppress the afterpulsing effect.

Timing jitter is usually measured using an instrument capable of time-correlated single-photon counting or a common time-to-digital converter. The detection signal in the quenching electronics and the synchronized output from the signal generator can be used as 'Start' and 'Stop' of the timing measurement instrument, respectively. After eliminating the intrinsic jitter due to the signal generator, the laser, and the timing measurement system, the timing jitter of the whole detector system can be obtained.

\section{LOW-FREQUENCY GATING}

Gated mode is a simple and effective approach to suppress DCR and afterpulses for synchronous single-photon detection. ${ }^{46}$ When the

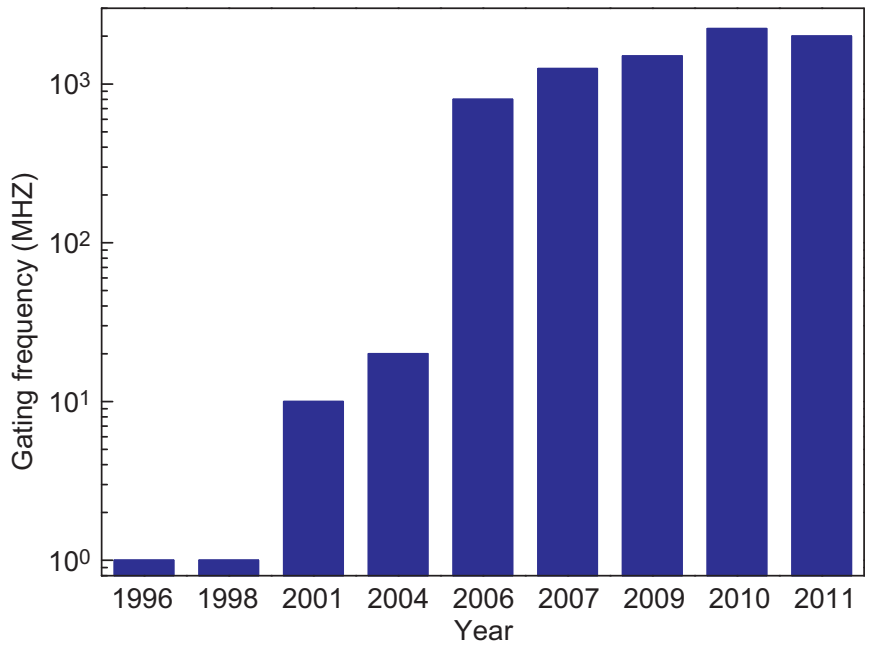

Figure 4 The evolution of gating frequency for InGaAs/InP SPADs. All the data are taken from the references. SPAD, single-photon avalanche diode.

electronic signals of the gates are coupled to a SPAD, derivative signals are created due to the capacitive response of the SPAD, and avalanche signals are superimposed on the derivative signals. Suppressing the derivative signals to effectively extract avalanche signals is the key task in the gated quenching electronics. The amplitudes of derivative signals depend on the rise (and fall) time of gates, gate amplitudes and quenching circuits. The amplitudes of avalanche signals depend on $V_{\mathrm{ex}}$ and $t_{\mathrm{w}}$. $t_{\mathrm{w}}$ is the most important parameter in the gated quenching scheme. If $t_{\mathrm{w}}$ cannot be too short, e.g. 1 ns or less, the afterpulsing contribution is still considerably high. To suppress the afterpulsing effect, a long hold-off time at the level of microseconds is necessary, which substantially limits the gating frequency. Using conventional gating techniques, the maximum frequency is limited to a few tens of $\mathrm{MHz}$.

The evolution of gating frequency is shown in Figure 4. Dramatic increases in gating frequency occurred during the dozen years between 1998 and 2010. In this Review, we define $100 \mathrm{MHz}$ as a critical point, which means that gating techniques with frequency below (above) $100 \mathrm{MHz}$ are categorized as low-frequency (high-frequency) gating.
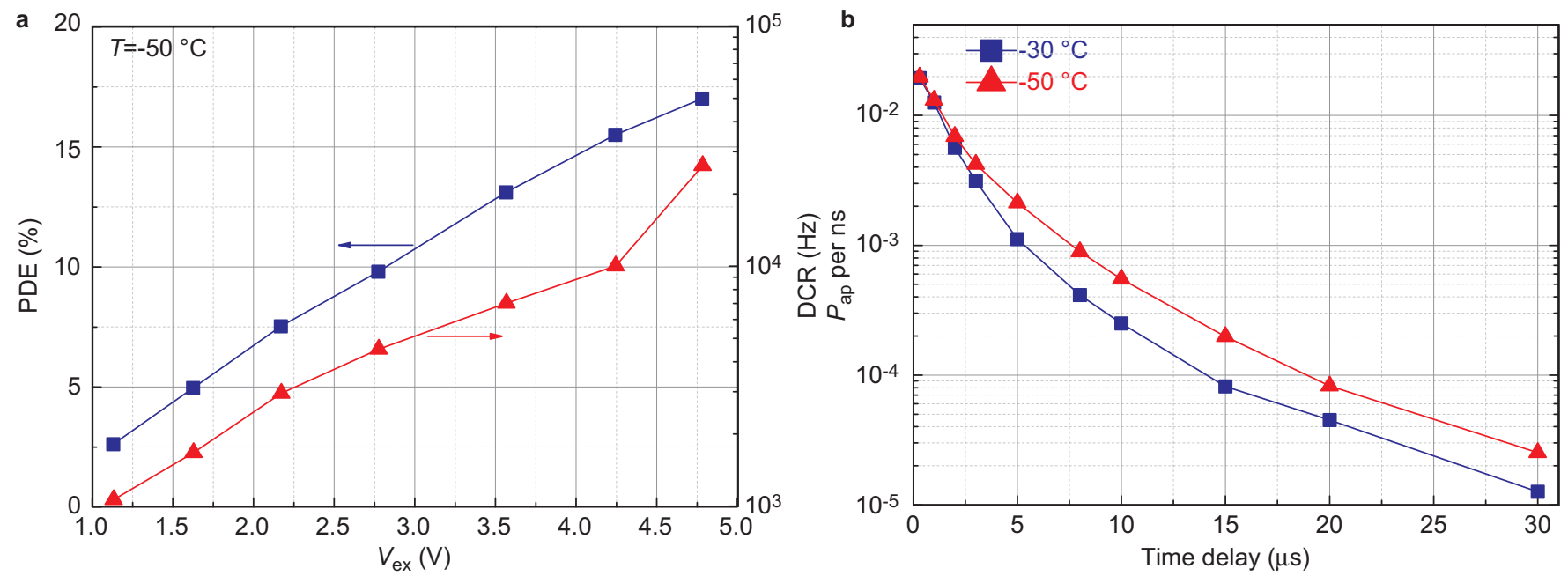

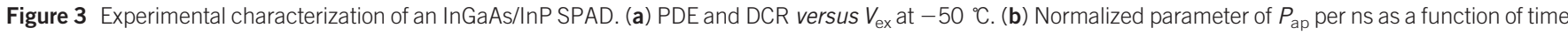

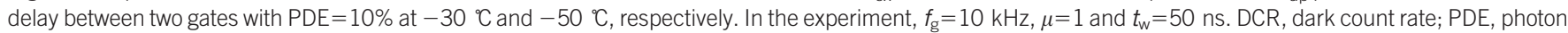
detection efficiency; SPAD, single-photon avalanche diode. 
In this section, we describe the development and evolution of lowfrequency gating, and introduce some representative gating techniques among numerous works ${ }^{46,51,54-75}$ in the past, which could be good references to invent new quenching techniques.

The coincidence method ${ }^{64}$ is a standard technique for avalanche extraction in low-frequency gating. Electronic gate signals, as shown in Figure $5 b(1)$, are alternating current (AC) coupled to the cathode of SPAD. Output signals with the superposition of avalanches and derivative signals at the anode of SPAD (Figure $5 \mathrm{~b}(2)$ ) are discriminated by a comparator. The comparator outputs (Figure $5 \mathrm{~b}(3)$ ) and the auxiliary signals synchronized to the gates (Figure $5 b(4)$ ) are inputs to an AND logic gate, whose outputs (Figure $5 \mathrm{~b}(5)$ ) are effective avalanche events. The timing of the auxiliary signals is precisely controlled to avoid the coincidence with the discrimination outputs of the derivative signals. The coincidence method can be easily implemented using analog and digital circuits. However, there are still some drawbacks in such a scheme. For instance, to avoid false detections due to electronic noise, the threshold of the comparator is necessarily increased. This may result in small avalanches not being detected, e.g., avalanches that have less time to build up because they occur near the end of gates. If the derivative signals are well suppressed, the amplitude ratio of avalanches to background signals can be effectively improved and hence, the comparator threshold can be further lowered.

Common-mode cancellation is the basic idea behind suppressing capacitive responses, and there are diverse approaches to cancelling the derivative signals. In 2000, Bethune and Risk ${ }^{57}$ reported a transient cancellation technique using radio frequency (RF) delay lines, and based on this detector, they implemented an autocompensating quantum cryptography system. ${ }^{57,59}$ Details about the detector can be found in Ref. 63.

The RF delay line scheme and its timing diagram are shown in Figure 6. Electronic pulses (Figure $6 \mathrm{~b}(1)$ ) are AC coupled to the cathode of the SPAD via a directional coupler and are also connected with an open-circuit cable whose length is $L$. The non-inverted reflection at the end of the open-circuit cable forms an additional gate pulse (Figure $6 \mathrm{~b}(2)$ ) delayed by a time interval of $2 L / v$ relative to the original pulse, where $v$ is the propagation speed of electromagnetic waves in the cable. The anode of the SPAD is connected to another cable whose length is the same as the upper cable, but a short-circuit termination of this second delay cable results in an inverted reflection. The transient signal at the anode is the superposition of the SPAD response signal due to the initial gate pulse and its non-inverted reflection from the upper cable along with its inverted reflection that is delayed by
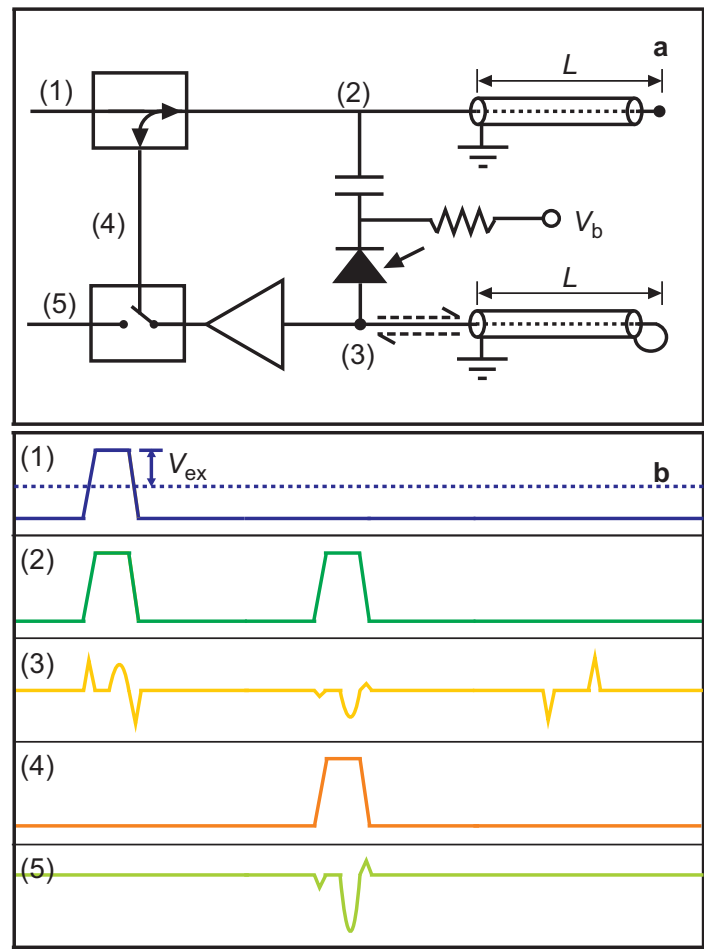

Figure 6 Scheme (a) and timing diagram (b) of RF delay line cancellation method for low-frequency gating. $V_{\mathrm{b}}$, bias voltage; $V_{\mathrm{ex}}$, excess bias; $L$, cable length.

$2 L / v$. As a result of this superposition, the parasitic derivative signals from the two reflections cancel each other, and with appropriate timing of photon arrivals the avalanche signal clearly stands out (Figure $6 \mathrm{~b}(3)$ ). After passing the controlling gate (Figure $6 \mathrm{~b}(4)$ ) and amplifier, the amplified avalanche signals (Figure $4 \mathrm{~b}(5)$ ) then can be easily discriminated. With effective cancellation, the discrimination threshold is substantially lowered, which allows for further reduction of the gating width. As a consequence of shorter gate, the afterpulsing performance of the SPAD is improved. The primary drawback of this approach is that the intrinsic delay due to the cable reflections severely limits the maximum gating frequency.

In 2002, Tomita et al. ${ }^{60}$ implemented a two-channel detector system using the double-SPAD technique. Two SPADs are operated as shown in Figure 7. These two SPADs have very similar semiconductor parameters such as diode capacitance and excess bias-efficiency relationship.
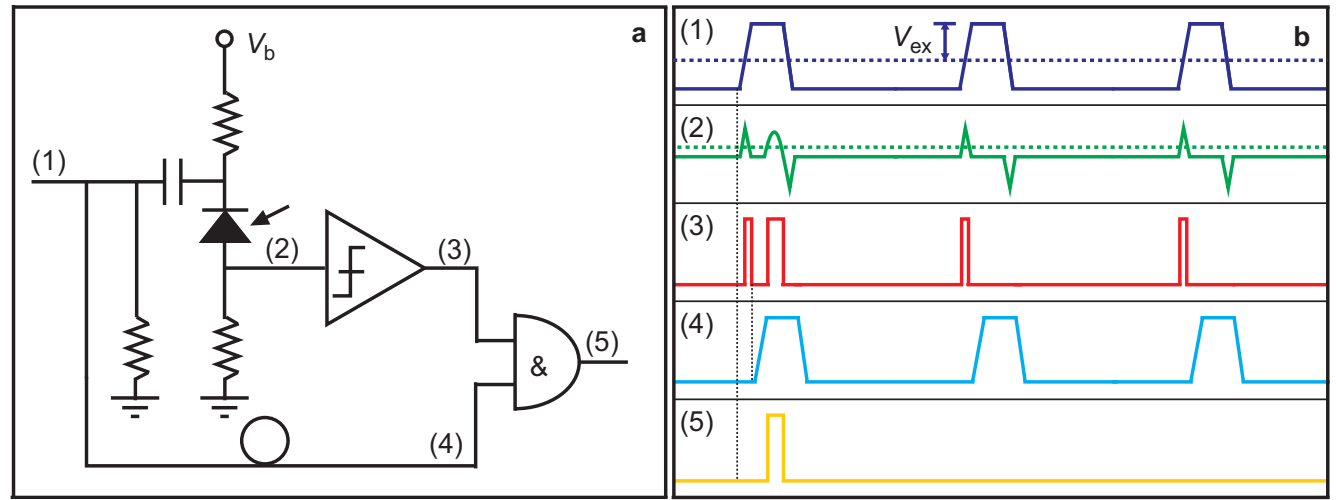

Figure 5 Scheme (a) and timing diagram (b) of coincidence method for avalanche extraction in low-frequency gating. $V_{\mathrm{b}}$ : bias voltage. $V_{\text {ex }}$ : excess bias. The signal amplitudes in this and the following figures are not to scale. 


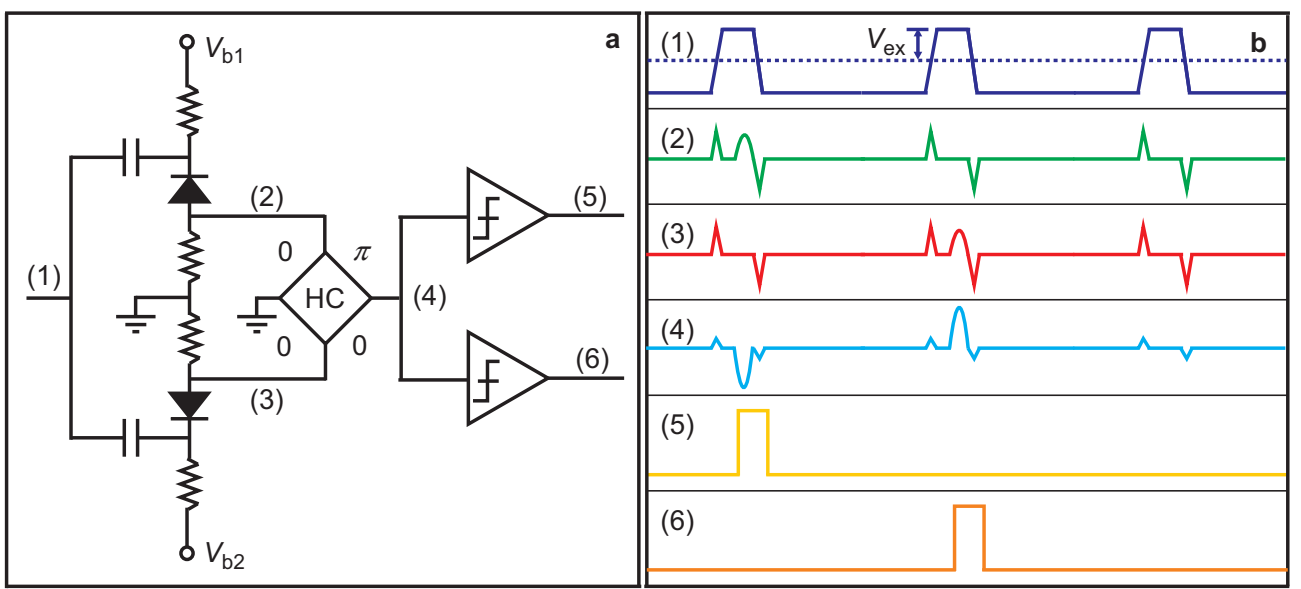

Figure 7 Scheme (a) and timing diagram (b) of double-SPAD cancellation method for low-frequency gating. $V_{\mathrm{b} 1}, V_{\mathrm{b} 2}$, bias voltages; $V_{\mathrm{ex}}$, excess bias. HC, hybrid coupler; SPAD, single-photon avalanche diode.

Electronic pulses (Figure $7 \mathrm{~b}(1)$ ) are AC coupled to the cathodes of both the SPADs in parallel. The anodes of SPADs are connected with a $180^{\circ}$ hybrid coupler (HC). Due to the similar capacitive responses, the derivative signal shapes at the anode of the upper SPAD (Figure $7 \mathrm{~b}(2)$ ) and that at the anode of the lower SPAD (Figure $7 \mathrm{~b}(3)$ ) are almost the same, given that avalanches of the two SPADs are created at different gates. After passing the HC, the derivative signals cancel each other. The avalanche of the upper SPAD is reversed and the avalanche of the lower SPAD remains the same polarity, as shown in Figure $7 \mathrm{~b}(4)$. The avalanches are then discriminated by two comparators with negative (Figure $7 \mathrm{~b}(5)$ ) and positive (Figure $7 \mathrm{~b}(6)$ ) thresholds, whose outputs indicate detection clicks at the upper and the lower SPADs, respectively.

This double-SPAD technique also has some drawbacks. The primary drawback is avalanche cancellation. When avalanches of the two SPADs are created at the same gate, the two avalanche signals will cancel each other due to the HC. Therefore, the two detectors cannot output detection clicks at the same time. For some specific applications, this drawback is still acceptable. When a single photon arrives at two-port devices such as beam splitter and polarizing beam splitter, the photon can only be detected by one of the SPADs. In a QKD system, this technique is well suited for the receiver integration. In addition, to maximize the suppression ratio for this scheme, selecting two SPADs with very similar parameters is necessary, which may be achieved with efforts of device screening in practice.

There are also some variant techniques to avoid the above drawbacks. For instance, using a common diode to replace one of the SPADs is a practical solution to avoid both the problems of avalanche cancellation and device screening at the cost of a potential decrease in the suppression ratio. The $\mathrm{HC}$ as shown in Figure 7 is the key component to eliminate the derivative signals. Besides the HC, there are some common devices that can implement the function of signal suppression such as common-mode choke coils, radiofrequency transformers and differential amplifiers. In low-frequency gating, these common devices may be used for practical implementations.

\section{HIGH-FREQUENCY GATING}

Increasing the gating frequency for SPADs is critical for applications requiring high count rate. Actually, the invention of high-frequency gating techniques was originally driven by high-rate QKD applications. For a point-to-point QKD system, the raw key rate $\left(R_{\text {raw }}\right)$ is roughly calculated as ${ }^{30} R_{\text {raw }} \propto \frac{1}{2} f \mu t \eta$, where $f$ is the system clock frequency that is usually the same as $f_{\mathrm{g}}$, $t$ is the channel transmission, and $\eta$ is detection efficiency. Given a QKD system operating at a certain distance, $t$ and $\eta$ are fixed. Also, for security consideration, $\mu$ cannot be simply increased. Therefore, increasing $f$ is the only way to achieve higher bit rate. ${ }^{30}$

As explained in the above sections, the primary obstacle of highfrequency gating is the afterpulsing effect. One of the most effective approaches for afterpulsing suppression is reducing $t_{\mathrm{w}}$. Hence the key technology challenge of high-frequency gating is weak avalanche extraction among strong stray signal when $t_{\mathrm{w}}$ is ultrashort. In 2006, Namekata et al. ${ }^{76}$ first reported a high-frequency gating detector with a clock rate of $800 \mathrm{MHz}$. The technique is called sine wave gating (SWG), as shown in Figure 8.

In this scheme, sine waves (Figure $8 \mathrm{~b}(1)$ ) with peak-peak amplitude of $V_{\mathrm{pp}}$ are used as gates. Since the frequency spectrum of an ideal sine wave is pure, the response signals are only composed of sine waves with the fundamental frequency and higher order harmonics. Avalanches are superimposed over the response signals (Figure $8 \mathrm{~b}(2)$ ). These response signals can be easily eliminated by cascaded band-stop filters at center frequencies of $f_{\mathrm{g}}, 2 f_{\mathrm{g}}, 3 f_{\mathrm{g}}$, etc. After the process of filteringamplification-filtering, weak avalanches can be finally extracted (Figure $8 \mathrm{~b}(3)$ ) and discriminated (Figure $8 \mathrm{~b}(4)$ ). Low-pass filters are normally used before the comparator in order to smooth the analog signals of amplified avalanches. ${ }^{76}$

When $f_{\mathrm{g}}$ is at the level of $\mathrm{GHz}, t_{\mathrm{w}}$ is generally very short and this parameter may be further reduced by tuning $V_{\mathrm{pp}}$. Ideally, $t_{\mathrm{w}}$ can be calculated as $t_{\mathrm{w}}=\left(1-\frac{2}{\pi} \arcsin \frac{2 V_{\mathrm{ex}}}{V_{\mathrm{pp}}}\right) / f_{\mathrm{g}}$. Empirically, in a $\mathrm{GHz}$ SWG detector system, $t_{\mathrm{w}}$ can be as short as around $200 \mathrm{ps}$, which drastically suppresses the afterpulsing effect and hence greatly improves the count rate. The maximum count rate of the SWG scheme can in principle reach the same value as $f_{\mathrm{g}}$. However, due to remaining afterpulsing contributions, a small hold-off time of tens of ns may still be necessary, which limits the maximum count rate to the range of tens of MHz. This is higher than low-frequency gating detectors, often by several orders of magnitude. Since the conventional hold-off time method cannot be directly applied to the SWG scheme, 'count-off time' is normally utilized instead. ${ }^{77}$

In 2007, Yuan et al. ${ }^{78}$ implemented a new high-frequency gating technique called self-differencing. As shown in Figure 9, square waves 


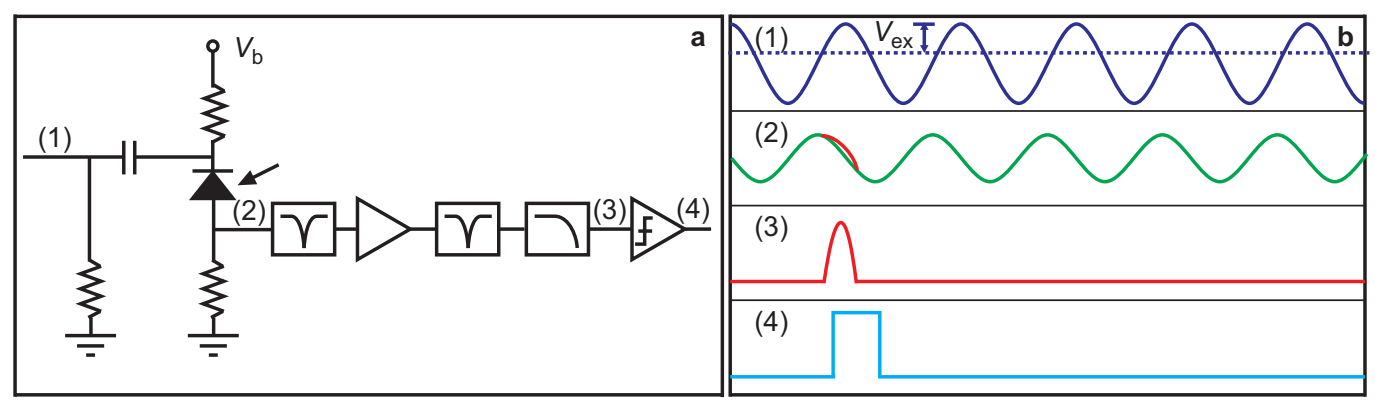

Figure 8 Scheme (a) and timing diagram (b) of sine wave gating for high-frequency gating. $V_{\mathrm{b}}$, bias voltage; $V_{\mathrm{ex}}$, excess bias.

with a clock rate of $\mathrm{GHz}$ (Figure $9 \mathrm{~b}(1)$ ) are coupled to the cathode of the SPAD. The response signals at the anode are first split by a power divider, and signals at one of output ports are delayed by one period. The two signals (Figure $9 \mathrm{~b}(2)$ and Figure $9 \mathrm{~b}(3)$ ) are then subtracted from each other. As a result, the derivative signals can be effectively eliminated. After amplification, avalanches (Figure $9 \mathrm{~b}(4)$ ) with positive and negative parts can be easily discriminated. The self-differencing method is similar to the double-SPAD technique introduced in the previous section. For practical implementation, the subtraction circuit can be achieved using devices such as RF transformers. Similarly, the drawback of avalanche cancellation also exists when avalanches occur in adjacent gates. Therefore, the maximum ideal count rate is only $f_{\mathrm{g}} / 2$.

Presently sine wave gating and self-differencing are the two fundamental techniques used for high-frequency single-photon detection. Each technique has its own advantages and disadvantages in practice. So far, many groups have already implemented high-frequency detector systems using these two techniques or improved schemes, ${ }^{76-94}$ and the clock rate has been increased to over $2 \mathrm{GHz}$.

In 2009, the Geneva group reported a practical solution for highfrequency gating by combining sine wave gating and self-differencing together. ${ }^{77}$ Sine waves were applied as gates, and filters were used to partly reduce response signals while a self-differencing circuit was used to finally eliminate response signals. In such a way, the suppression requirement for the filtering circuit and the self-differencing circuit was moderate compared to that of using each technique individually. ${ }^{77} \mathrm{Wu}$ et al. $^{95}$ demonstrated an optical selfdifferencing method. The response signals of the SPAD were used to drive a laser diode after amplification, and then the optical pulses emitted from the laser diode with the same shapes as the response signals were divided by an optical beam splitter. One output port of the beam splitter was delayed by one period, and two channels of optical pulses were coupled to two balanced photodiodes for cancellation, respectively.
In 2010, the Geneva group presented a SWG detector with a clock rate of $2.23 \mathrm{GHz}{ }^{82}$ This frequency is the fastest reported so far and approaches the bandwidth limit of current commercial InGaAs/InP SPADs. Applying such detectors to QKD applications, simulations show that the detector performance is already comparable to a common SNSPD, and the maximum communication distance can reach around $200 \mathrm{~km} .{ }^{82}$ Chen et al. ${ }^{84}$ invented an improved gating technique called double self-differencing. This cascaded self-differencing circuit could more effectively reduce response signals and thus further improve the SNR of avalanches.

In 2012, Liang and co-workers ${ }^{88}$ first developed a stand-alone instrument consisting of a fully integrated SPD system with 1.25 GHz sine wave gating. The $2 \mathrm{U}$ rack instrument included diverse functionalities such as precise controls for temperature, bias, amplitude, comparator threshold, delay, and friendly user-interface and relevant auxiliary hardware interfaces. Walenta et al. ${ }^{89}$ also implemented a $1.25 \mathrm{GHz}$ SWG detector module using only low-pass filters, which was well suited for QKD system integration. Similar work has also been done by Liang et al. ${ }^{87}$ and based on the $1 \mathrm{GHz}$ gating detector, they demonstrated a laser ranging experiment. ${ }^{85}$

Apart from sine waves and square waves, in 2013, Zhang et al. ${ }^{93}$ demonstrated a high-frequency gating detector using Gaussian pulses. Given time-dependent Gaussian pulses $G(t)=\frac{1}{\sqrt{2 \pi} \sigma} e^{-t^{2} / 2 \sigma^{2}}$, where $\sigma$ is related to pulse width, its derivative signals can be written as $\frac{\mathrm{d} G(t)}{\mathrm{d} t}=\frac{-t}{\sqrt{2 \pi} \sigma^{3}} e^{-t^{2} / 2 \sigma^{2}}$. The exponential term in the derivative is the same as that in $G(t)$. The falling edge shape of the bipolar response signals is similar to that of the original Gaussian pulses. If this falling edge is combined with the rising edge of a referencing attenuated and phase-matched Gaussian pulses which are synchronized to the original one, the falling edge can be cancelled and a steady plateau is formed. Avalanches are superimposed onto the plateau and hence, can be easily discriminated.
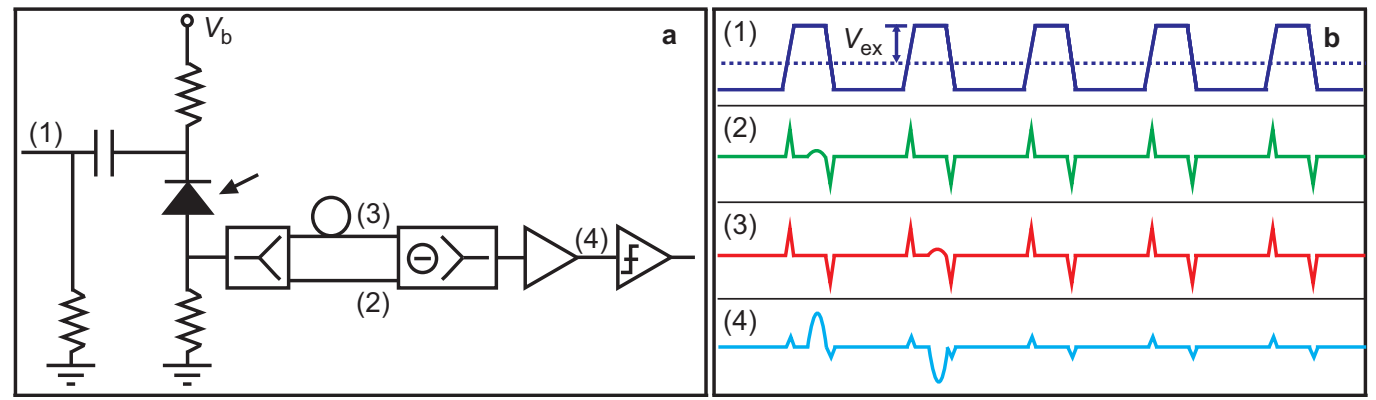

Figure 9 Scheme (a) and timing diagram (b) of self-differencing for high-frequency gating. $V_{b}$, bias voltage; $V_{\text {ex }}$, excess bias. 


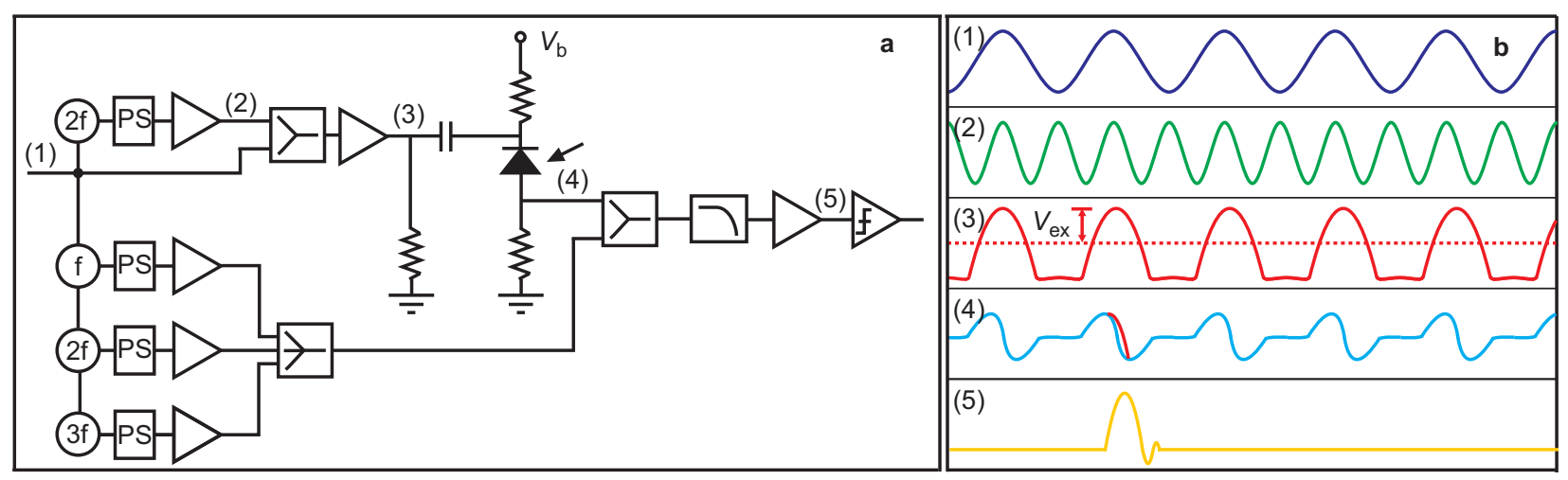

Figure 10 Scheme (a) and timing diagram (b) of harmonics subtraction for high-frequency gating. $V_{\mathrm{b}}$, bias voltage; $V_{\mathrm{ex}}$, excess bias. PS, phase shifter.

Recently, Restelli et al. ${ }^{94}$ used harmonics subtraction instead of band-stop filters for SWG, which could bring better afterpulsing performance compared with the standard filtering method. The harmonics subtraction method is shown in Figure 10. A sine wave (Figure $10 \mathrm{~b}(1)$ ) and its second harmonic (Figure $10 \mathrm{~b}(2)$ ) are combined and then amplified up to $20 \mathrm{~V}$ peak-to-peak (Figure $10 \mathrm{~b}(3)$ ). Although the amplified gates are distorted, the rising and falling edges are steeper than those of the fundamental sine wave. High amplitude and steep slope can further shorten the gating width compared with the standard SWG scheme. The first, second and third harmonics are synthesized with phase and amplitude control and then combined with the response signals (Figure $10 \mathrm{~b}(4)$ ) at a power divider. As a result, frequency components due to the harmonics are removed so that remaining avalanches are easily extracted after passing through a low-pass filter and a low-noise amplifier (Figure 10b(5)).

SPADs are traditionally believed to output only click or non-click for incident photons, but not to resolve photon numbers. However, with the high-frequency gating techniques SPADs may have the capability of PNR. Owing to ultra-short avalanche duration, weak avalanches are always in the sub-saturation stage so that different numbers of photon-generated carriers can result in different avalanche amplitudes. Experiments of PNR using high-frequency gating SPADs have been demonstrated. ${ }^{95-97}$ However, the incident photon numbers, cannot be effectively resolved, due to the low detection efficiency.

In all the above high-frequency gating schemes, InGaAs/InP SPADs have been used. However, these techniques can be widely applied for other SPADs of different materials. For instance, photon number resolving ${ }^{97}$ and high efficiency ${ }^{98}$ Silicon SPADs using high-frequency gating techniques have been reported.

\section{FREE-RUNNING OPERATIONS}

Passive quenching ${ }^{16}$ is the fundamental approach for free-running single-photon detection, ${ }^{43}$ and this scheme was initially demonstrated about two decades ago. However, there are still two technical problems to be solved. One is long recovery time and the associated baseline shift due to avalanche pileups during recovery. The other is large afterpulse probability. The recovery problem could be overcome using a resetting circuit in practice. Thus, the afterpulsing problem remains the key challenge in implementing free-running detectors. Simply increasing the hold-off time severely limits the count rate. Some groups reported different feasible methods to reduce the afterpulsing contribution. Warburton et al. ${ }^{99}$ implemented a free-running InGaAs/InP SPAD through carefully optimizing the operation conditions such as lowering excess bias and increasing temperature. The Virginia group implemented a sophisticated method for the afterpulsing reduction, ${ }^{100-102}$ i.e., removing the package of device and connecting the contacts of the SPAD and the quenching circuit by chip-to-chip wire bonding. In such a way, the stray capacitance was minimized.

Apart from passive quenching, free-running SPADs using active quenching have also been reported. In such cases, reducing the quenching time was crucial for the afterpulsing suppression. The Geneva group implemented a free-running detector based on an active quenching ASIC to minimize the parasitic capacitance of the electronics. ${ }^{103}$

High-frequency gating can also be used to mimic free-running operation, and relevant experiments for applications have been demonstrated. ${ }^{85,104}$ The advantages of this method are low afterpulsing probability and high count rate, while the disadvantage is photon loss due to the duty cycle. For instance, given a gating frequency of $1 \mathrm{GHz}$ and a gating width of $200 \mathrm{ps}$, the equivalent detection efficiency in the free-running mode is only $1 / 5$ of that in the gating mode.

In this section, we will focus on the recent progress of free-running detectors including passive quenching and active reset (PQAR) and negative feedback avalanche diodes (NFADs).

Figure 11 shows a typical PQAR scheme implemented by the Virginia group. ${ }^{101}$ The large resistor in the standard passive quenching schemes is replaced by a high-frequency GaAs FET. The FET is appropriately biased to hold the off-state. In the off-state, the FET has high impedance so that avalanches can be quenched passively. The avalanche signals are direct current coupled to a low-noise amplifier at the cathode of the SPAD. The discrimination outputs of the amplified avalanches are used to drive a pulse generator, which creates reset

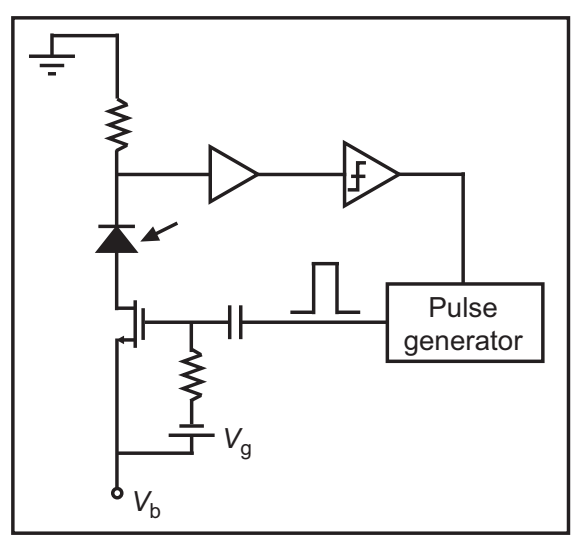

Figure 11 Typical scheme of passive quenching and active reset for freerunning SPADs. $V_{\mathrm{b}}$, bias voltage; $V_{\mathrm{g}}$, direct current bias to the gate terminal. SPAD, single-photon avalanche diode. 
signals after the period of the hold-off time. The reset signals are AC coupled to the gate terminal of the FET to activate the FET into the onstate. In the on-state, the FET has low impedance so that the voltage at the anode of the SPAD can be rapidly recharged to the initial value $\left(V_{\mathrm{b}}\right)$. This PQAR scheme offers good dynamic range performance.

Recently a new kind of SPAD device, i.e., NFAD, was invented. ${ }^{105-110}$ The NFAD monolithically integrates a high-resistance thin film resistor with the semiconductor structure, as shown in Figure 12a. With this design, the NFAD exploits passive quenching. Due to the integration, the parasitic capacitance of the quenching circuit is minimized so that the afterpulsing performance of the NFAD is significantly improved compared with the common SPAD. In the equivalent circuit of the NFAD (Figure 12b), integrated resistor $\left(R_{\mathrm{L}}\right)$ is larger than diode equivalent impedance $\left(R_{\mathrm{d}}\right)$ by several orders of magnitude. Therefore, avalanches can be quenched swiftly due to the voltage drop on $R_{\mathrm{L}}$. When the NFAD is recharging, the recovery time is determined by $R_{\mathrm{L}} C_{\mathrm{d}}$. The quenching electronics of the NFAD can be similar to that of PQAR circuits, as shown in Figure 12c. Avalanches are AC coupled out. After amplification and discrimination, the outputs are driven by a hold-off time circuit. The amplified hold-off time signals are DC coupled to the anode of the NFAD. When hold-off time signals are at a high level, the bias of the NFAD is below $V_{\mathrm{b}}$ due to the voltage lift at the anode of the NFAD. Using such quenching electronics, Lunghi et al. ${ }^{111}$ demonstrated an NFAD-based detector system with a detection efficiency of $10 \%$ and a DCR of $600 \mathrm{~Hz}$ at $-50{ }^{\circ} \mathrm{C}$.

In 2012, Yan et al. ${ }^{112}$ implemented a similar free-running detector using the NFAD in which a transformer was used as an avalanche readout circuit. At $-80{ }^{\circ} \mathrm{C}$, a detection efficiency of $10 \%$ and a DCR of $100 \mathrm{~Hz}$ was achieved. Using a Stirling cooler, the Geneva group significantly improved the noise performance of the free-running detector. ${ }^{113}$ At $-110{ }^{\circ} \mathrm{C}$, with a detection efficiency of $10 \%$, DCR was reduced down to a record level of only $1 \mathrm{~Hz}$, and $P_{\text {ap }}$ was $2.2 \%$ for $20 \mu$ s hold-off time. Such impressive performance implies that NFADs could be comparable to SNSPDs except for the limited count rates. In particular, these detectors are the ideal choice for longdistance QKD, where the limitation in count rate is not an issue.

\section{QUANTUM COMMUNICATION APPLICATIONS}

InGaAs/InP SPADs have been widely used for optical fiber quantum communication, what we would like to illustrate in this section. Particularly in QKD, progress in transmission distance has been since ever closely linked to the progress in the development of low-noise SPDs. InGaAs/InP SPADs, in the low-frequency gating mode, were used first for QKD at the end of the 1990s using auto-compensating plug-and-play systems. ${ }^{114}$ In 1999 , Bourennane et al. ${ }^{115}$ performed a plug-and-play experiment with a fiber transmission distance of $40 \mathrm{~km}$. Hughes et al. ${ }^{116}$ realized one-way phase-encoding QKD implementing both the BB84 and B92 protocols over $48 \mathrm{~km}$ of optical fiber. In 2002, Stucki et al. ${ }^{117}$ reported a field QKD experiment over $67 \mathrm{~km}$ installed fiber between Geneva and Lausanne. Then Kosaka et al. ${ }^{118}$ increased the QKD distance up to $100 \mathrm{~km}$ with InGaAs/InP singlephoton detectors using a double-SPAD scheme as introduced previously. In 2004, the Toshiba group further increased the QKD distance up to $122 \mathrm{~km}$ using low DCR SPADs. ${ }^{119}$ All the above QKD experiments used weak laser pulses (coherent states) to mimic a singlephoton source. However, due to the photon-number-splitting attack, the secure distance was significantly limited. Thanks to the decoy-state scheme subsequently proposed, this primary obstacle was completely eliminated so that practical applications of QKD could be possible. In 2007, Peng et al. ${ }^{120}$ first demonstrated a decoy-state QKD experiment over $100 \mathrm{~km}$ using InGaAs/InP SPADs.

Longer distances and higher rates were achieved using highfrequency gating featuring less afterpulsing. In 2007, Namekata et al. ${ }^{121}$ first implemented a differential phase shift QKD experiment using SWG SPADs. At a clock rate of $500 \mathrm{MHz}$, the final secure key rate reached $0.33 \mathrm{Mbps}$ over a distance of $15 \mathrm{~km}$. The same group then implemented $24 \mathrm{kbps}$ secure key rate over $100 \mathrm{~km}$ fiber distance ${ }^{122}$ by increasing the clock rate up to $2 \mathrm{GHz}$ and optimizing the SPAD performance with an ultralow dark count probability per gate of $2.8 \times 10^{-8}$ at a detection efficiency of $6 \%$. In 2008, Yuan et al. ${ }^{123}$ demonstrated a $\mathrm{GHz}$ QKD experiment using self-differencing SPADs and achieved $27.9 \mathrm{kpbs}$ secure key rate at $65.5 \mathrm{~km}$. Then the Toshiba group further improved both the bit rate and the system stability to push forward GHz QKD system for practical uses. ${ }^{124}$ In 2010, they demonstrated $1 \mathrm{Mbps}$ bit rate at $50 \mathrm{~km}$ over a continuous operation of $36 \mathrm{~h}^{125}$ In 2014, they performed the coexistence experiment of GHz QKD with classical optical communication, ${ }^{126}$ in which quantum data and bidirectional 10 Gbps classical data were combined in a single fiber using dense wavelength division multiplexing. The secure key rate reached $2.38 \mathrm{Mbps}$ over $35 \mathrm{~km}$ and the fiber distance could be extended up to $70 \mathrm{~km}$.

Due to the very short gates and hold-off times, dark count probabilities per gate are becoming pretty low and the afterpulsing may become the main noise contribution for QKD. For this reason the optimal operation temperature of SPADs can be close to room temperature, which is very convenient for commercial systems. ${ }^{89,127}$

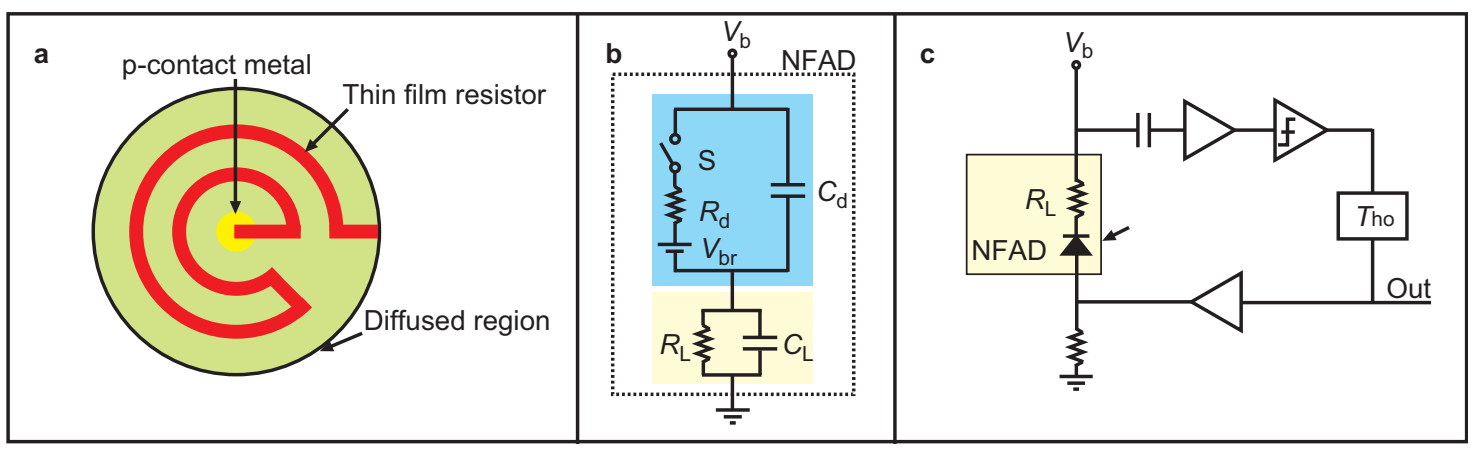

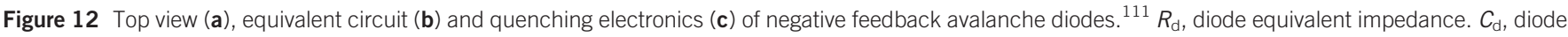

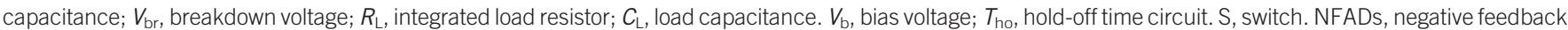
avalanche diodes. 
For even longer distances, unpractical SNSPDs are generally employed. However, very recently secure QKD over more than $300 \mathrm{~km}$ of fiber have been realized, using ultra-low noise free-running NFADs. ${ }^{128}$

Apart from weak coherent states, entanglement distribution is another approach to implement QKD. In 2004, the Geneva group implemented the distribution of time-bin entangled photon pairs over $50 \mathrm{~km}$ fiber. ${ }^{129}$ Takesue demonstrated experimental distribution of time-bin entanglement generated using spontaneous four-wave mixing over $60 \mathrm{~km} .{ }^{130}$ In 2007, the distribution of polarization entanglement over $100 \mathrm{~km}$ was achieved by the Vienna group. ${ }^{131}$ Finally, Dynes et al. ${ }^{132}$ demonstrated distribution of time-bin entangled photons over a record distance of $200 \mathrm{~km}$ using self-differencing InGaAs/InP SPADs.

Except QKD, other quantum communication protocols have also been implemented in optical fiber. The Geneva group demonstrated quantum teleportation over $2 \mathrm{~km}$ of telecom fiber ${ }^{133,134}$ and also in an installed fiber network. ${ }^{135}$ In 2008, Bogdanski et al. ${ }^{136}$ reported a QSS experiment for five parties based on a single-qubit protocol. In 2013, Ma et al. ${ }^{137}$ increased the fiber distance up to $50 \mathrm{~km}$ for single-qubit QSS, and circular QSS was also demonstrated in fiber. ${ }^{138}$ Counterfactual quantum cryptography was proposed in $2009^{29}$ and experimentally demonstrated by Ren et al. ${ }^{139}$ and Liu et al. ${ }^{140}$

\section{CONCLUSIONS AND OUTLOOKS}

III-V SPADs are the most practical tools for ultra-weak light detection in the near-infrared. In the past decades, both the academic and industrial communities have made great efforts to achieve performance improvements of SPADs. In the field of semiconductor devices, dedicated devices for single-photon detection are designed and fabricated, while APDs designed for classical optical communication are no longer widely used in photon counting applications. The device performance of the SPAD itself has been gradually improved. Also, new devices like NFADs and self-quenching SPADs ${ }^{141,142}$ have appeared, which may particularly improve the performance of certain parameters and alleviate the requirements of quenching electronics. In the field of quenching electronics, diverse techniques have been invented for both gating and free-running operations. The gating frequency has been increased up to $2 \mathrm{GHz}$ and relevant techniques have been quickly applied to QKD. In this review, we have surveyed the technical advances in lowfrequency gating, high-frequency gating and free-running operation, and we have described some representative quenching schemes.

In the future, the development and evolution of SPAD-based nearinfrared detectors will be continuously pushed forward in the same way. On the one hand, the SPADs themselves should have better device-level performance. This requires research and focused efforts addressing various device attributes such as structure design and optimization, high-quality material growth and fabrication technology. The key parameters to be considered in device design include PDE, DCR and $P_{\mathrm{ap}}$. PDE and DCR are the intrinsic parameters and are generally independent from quenching electronics, which means that the parameter performance cannot be improved using different electronics at the same operating conditions. Thus, high PDE and low DCR are the two core objectives in future device design. Shrinking the size of the SPAD could be an effective approach for the reduction of DCR and $P_{\text {ap }}$, and relevant investigations have started. However, fiber coupling could be a crucial technology challenge for small-size SPADs. Another benefit of improvements in the DCR versus PDE tradeoff is operation at higher PDE given the same DCR performance. Apart from III-V SPADs, silicon-based devices could also be potential candidates for single-photon detection in the future-e.g., Ge/Si and InGaAs/Si-although currently, the DCR of these devices are still high compared with III-V SPADs.

On the other hand, quenching electronics is also important for practical detector systems. Therefore, inventing new quenching techniques and continuously optimizing the present quenching techniques are the fundamental tasks for the single-photon community. For these mature techniques, developing integrated circuits for quenching electronics is the future trend due to various advantages including cost-effectiveness, detector miniaturization, parasitic capacitance minimization and power reduction. For Si SPADs, such integrated circuits were achieved over a decade ago. ${ }^{14-146}$ Also, integrated quenching electronics is favorable for SPAD arrays. Due to both the improvement of device performance and the integration of readout circuits, in the future, developing practical III-V SPAD arrays is possible and significant for applications requiring multi-pixel near-infrared single-photon detection.

\section{ACKNOWLEDGEMENTS}

We acknowledge Wen-Hao Jiang for technical assistance. This work has been financially supported by the National Basic Research Program of China (Grant No. 2013CB336800), the National High-Tech R\&D Program (Grant No. 2011AA010802), the National Natural Science Foundation of China (Grant No. 61275121) and the Innovative Cross-disciplinary Team Program of CAS. HZ acknowledges the financial support from the Swiss NCCR QSIT.

1 Hadfield RH. Single-photon detectors for optical quantum information applications. Nat Photonics 2009; 3: 696-705.

2 Eisaman MD, Fan J, Migdall A, Polyakov SV. Invited Review Article: Single-photon sources and detectors. Rev Sci Instrum 2011; 82: 071101.

3 Gansen EJ, Rowe MA, Greene MB, Rosenberg D, Harvey TE et al. Photon-numberdiscriminating detection using a quantum-dot, optically gated, field-effect transistor. Nat Photonics 2007; 1: 585-588.

4 Blakesley JC, See P, Shields AJ, KardynałBE, Atkinson P et al. Efficient single photon detection by quantum dot resonant tunneling diodes. Phys Rev Lett 2005; 94: 067401.

5 Albota MA, Wong FNC. Efficient single-photon counting at $1.55 \mu \mathrm{m}$ by means of frequency upconversion. Opt Lett 2004; 29: 1449-1451.

6 Thew RT, Zbinden H, Gisin N. Tunable upconversion photon detector. Appl Phys Lett 2008; 93: 071104.

7 Shentu GL, Pelc JS, Wang XD, Sun QC, Zheng MY et al. Ultralow noise up-conversion detector and spectrometer for the telecom band. Opt Express 2013; 21: 1398613991

8 Photomultiplier Tubes: Basics and Applications. 3rd ed. Hamamatsu: Hamamatsu Photonics; 2006

9 Goltsman GN, Okunev O, Chulkova G, Lipatov A, Semenov A et al. Picosecond superconducting single photon optical detector. Appl Phys Lett 2001; 79: 705-707.

10 Rosenberg D, Lita AE, Miller AJ, Nam SW. Noise-free high-efficiency photon-numberresolving detectors. Phys Rev A 2005; 71: 061803(R).

11 Peacock A, Verhoeve P, Rando N, Dordrecht A, Taylor BG et al. Single optical photon detection with a superconducting tunnel junction. Nature 1996; 381: 135-137.

12 Marsili F, Verma VB, Stern JA, Harrington S, Lita AE et al. Detecting single infrared photons with 93\% system efficiency. Nat Photonics 2013; 7: 210-214.

13 Itzler MA, Ben-Michael R, Hsu CF, Slomkowski K, Tosi A et al. Single photon avalanche diodes (SPADs) for $1.5 \mu \mathrm{m}$ photon counting applications. J Mod Opt 2007; 54: 283-304.

14 Jiang X, Itzler MA, Ben-Michael R, Slomkowski K. InGaAsP-InP avalanche photodiodes for single photon detection. IEEE J Sel Top Quantum Electron 2007; 13: 895-905.

15 Itzler MA, Jiang X, Entwistle M, Slomkowski K, Tosi A et al. Advances in InGaAsP based avalanche diode single photon detectors. J Mod Opt 2011; 58: 174-200.

16 Cova S, Ghioni M, Lacaita A, Samori C, Zappa F. Avalanche photodiodes and quenching circuits for single-photon detection. App/ Opt 1996; 35: 1956-1976.

17 Tosi A, Dalla Mora A, Zappa F, Cova S. Single-photon avalanche diodes for the nearinfrared range: detector and circuit issues. J Mod Opt 2009; 56: 299-308.

18 Gallivanoni A, Rech I, Ghioni M. Progress in quenching circuits for single photon avalanche diodes. IEEE Trans Nucl Sci 2010; 57: 3815-3826.

19 Bennett $\mathrm{CH}$, Brassard G. Quantum cryptography: public key distribution and coin tossing. In: Proceedings of International Conference on Computers, Systems and Signal Processing; 8-11 October 1984; Port Chester, New York, USA. IEEE: New York, USA, 1984, pp. 175-179. 
20 Ekert AK. Quantum cryptography based on Bell's theorem. Phys Rev Lett 1991; 67: 661-663.

21 Bennett CH, Brassard G, Crépeau C, Jozsa R, Peres A et al. Teleporting an unknown quantum state via dual classical and Einstein-Podolsky-Rosen channels. Phys Rev Lett 1993; 70: 1895-1899.

22 Hillery M, Bužek V, Berthiaume A. Quantum secret sharing. Phys Rev A 1999; 59: 1829-1834.

23 Cleve R, Gottesman D, Lo HK. How to share a quantum secret. Phys Rev Lett 1999; 83: 648-651.

24 Xiao L, Long GL, Deng FG, Pan JW. Efficient multiparty quantum-secret-sharing schemes. Phys Rev A 2004; 69: 052307

25 Long GL, Liu XS. Theoretically efficient high-capacity quantum-key-distribution scheme. Phys Rev A 2002; 65: 032302.

26 Boström K, Felbinger T. Deterministic secure direct communication using entanglement. Phys Rev Lett 2002; 89:187902.

27 Deng FG, Long GL, Liu XS. Two-step quantum direct communication protocol using the Einstein-Podolsky-Rosen pair block. Phys Rev A 2003; 68: 042317.

28 Deng FG, Long GL. Secure direct communication with a quantum one-time pad. Phys $\operatorname{Rev} A$ 2004; 69: 052319

29 Noh TG. Counterfactual quantum cryptography. Phys Rev Lett 2009; 103: 230501.

30 Gisin N, Ribordy G, Tittel W, Zbinden H. Quantum cryptography. Rev Mod Phys 2002; 74: 145

31 Scarani V, Bechmann-Pasquinucci H, Cerf NJ, Dušek M, Lütkenhaus $\mathrm{N}$ et al. The security of practical quantum key distribution. Rev Mod Phys 2009; 81: 1301-1350.

32 Gisin N, Thew R. Quantum communication. Nat Photonics 2007; 1: 165-171.

33 Yuan ZS, Bao XH, Lu CY, Zhang J, Peng CZ et al. Entangled photons and quantum communication. Phys Rep 2010; 497: 1-40.

34 Renker D, Lorenz E. Advances in solid state photon detectors. J Instrum 2009; 4: P04004

35 Campbell JC, Demiguel S, Ma F, Beck A, Guo X et al. Recent advances in avalanche photodiodes. IEEE J Sel Topics Quantum Electron 2004; 10: 777-787.

36 Campbell JC. Recent advances in telecommunications avalanche photodiodes. J Lightw Technol 2007; 25: 109-121.

37 Donnelly JP, Duerr EK, McIntosh KA, Dauler EA, Oakley DC et al. Design considerations for 1.06- $\mu \mathrm{m}$ InGaAsP-InP Geiger-mode avalanche photodiodes. IEEE J Quantum Electron 2006; 42: 797-809.

38 Pellegrini S, Warburton RE, Tan LJJ, Ng JS, Krysa AB et al. Design and performance of an InGaAs-InP single-photon avalanche diode detector. IEEE J Quantum Electron 2006; 42: 397-403.

39 Acerbi F, Anti M, Tosi A, Zappa F. Design criteria for InGaAs/InP single-photon avalanche diode. IEEE Photonics J 2013; 5: 6800209.

40 Ramirez DA, Hayat MM, Karve G, Campbell JC, Torres SN et al. Detection efficiencies and generalized breakdown probabilities for nanosecond-gated near infrared singlephoton avalanche photodiodes. IEEE J Quantum Electron 2006; 42: 137-145.

41 Hayat MM, Saleh BEA, Teich MC. Effect of dead space on gain and noise of doublecarrier multiplication avalanche photodiodes. IEEE Trans Electron Dev 1992; 39: 546-552.

42 Itzler MA, Jiang X, Ben-Michael R, Nyman B, Slomkowski K. Single photon avalanche diodes for near-infrared photon counting. Proc SPIE 2008; 6900: 69001E.

43 Rarity JG, Wall TE, Ridley KD, Owens PCM, Tapster PR. Single-photon counting for the 1300-1600-nm range by use of Peltier-cooled and passively quenched InGaAs avalanche photodiodes. Appl Opt 2000; 39: 6746-6753.

44 Ghioni M, Cova S, Zappa F, Samori C. Compact active quenching circuit for fast photon counting with avalanche photodiodes. Rev Sci Instrum 1996; 67: 3440.

45 Acerbi F, Frera AD, Tosi A, Zappa F. Fast active quenching circuit for reducing avalanche charge and afterpulsing in InGaAs/InP single-photon avalanche diode. IEEE J Quantum Electron 2013: 49. 563-569.

46 Ribordy G, Gautier JD, Zbinden H, Gisin N. Performance of InGaAs/InP avalanche photodiodes as gated-mode photon counters. Appl Opt 1998; 37: 2272-2277.

47 Karve G, Wang S, Ma F, Li X, Campbell JC et al. Origin of dark counts in In ${ }_{0.53} \mathrm{Ga}_{0.47} \mathrm{As} /$ $\mathrm{In}_{0.52} \mathrm{Al}_{0.48} \mathrm{As}$ avalanche photodiodes operated in Geiger mode. Appl Phys Lett 2005; 86: 063505

48 Cova S, Lacaita A, Ripamonti G. Trapping phenomena in avalanche photodiodes on nanosecond scale. IEEE Electron Dev Lett 1991; 12: 685-687.

49 Jiang X, Itzler MA, Ben-Michael R, Slomkowski K, Krainak MA et al. Afterpulsing effect in free-running InGaAsP single-photon avalanche diodes. IEEE J Quantum Electron 2008; 44: 3-11.

50 Eraerds P, Pomarico E, Zhang J, Sanguinetti B, Thew R et al. 32 bin near-infrared time-multiplexing detector with attojoule single-shot energy resolution. Rev Sci Instrum 2010; 81: 103105

51 Zhang J, Thew R, Gautier JD, Gisin N, Zbinden H. Comprehensive characterization of InGaAsP-InP avalanche photodiodes at $1550 \mathrm{~nm}$ with an active quenching ASIC. IEEE J Quantum Electron 2009; 45: 792-799.

52 Lunghi T, Korzh B, Sanguinetti B, Zbinden H. Absolute calibration of fiber-coupled single-photon detector. Opt Express 2014; 22: 18078-18092.

53 Ware M, Migdall A. Single-photon detector characterization using correlated photons: the march from feasibility to metrology. J Mod Opt 2004; 51: 1549-1557.

54 Lacaita A, Zappa F, Cova S, Lovati P. Single-photon detection beyond $1 \mu \mathrm{m}$ : performance of commercially available InGaAs/InP detectors. Appl Opt 1996; 35: 2986-2996.

55 Spinelli A, Davis LM, Dautet $\mathrm{H}$. Actively quenched single-photon avalanche diode for high repetition rate time-gated photon counting. Rev Sci Instrum 1996; 67: 55.
56 Hiskett PA, Buller GS, Loudon AY, Smith JM, Gontijo I et al. Performance and design

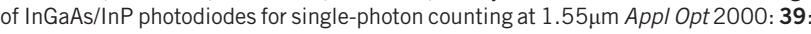
6818-6829.

57 Bethune DS, Risk WP. An autocompensating fiber-optic quantum cryptography system based on polarization splitting of light. IEEE J Quantum Electron 2000; 36: 340-347.

58 Stucki D, Ribordy G, Stefanov A, Zbinden H, Rarity JG et al. Photon counting for quantum key distribution with peltier cooled InGaAs/InP APDs. J Mod Opt 2001; 48: 1967-1981.

59 Bethune DS, Risk WP. Autocompensating quantum cryptography. New J Phys 2002; 4: 42.1 .

60 Tomita A, Nakamura K. Balanced, gated-mode photon detector for quantum-bit discrimination at $1550 \mathrm{~nm}$. Opt Lett 2002; 27: 1827-1829.

61 Namekata N, Makino Y, Inoue S. Single-photon detector for long-distance fiber-optic quantum key distribution. Opt Lett 2002; 27: 954-956.

62 Karve G, Zheng X, Zhang X, Li X, Ning $L$ et al. Geiger mode operation of an $\operatorname{In}_{0.53} \mathrm{Ga}_{0.47} \mathrm{As}-\mathrm{In}_{0.52} \mathrm{Al}_{0.48} \mathrm{As}$ avalanche photodiode. IEEE J Quantum Electron 2003; 39: 1281-1286.

63 Bethune DS, Risk WP, Pabst GW. A high-performance integrated single-photon detector for telecom wavelengths. J Mod Opt 2004; 51: 1359-1368.

64 Ribordy G, Gisin N, Guinnard O, Stucki D, Wegmuller M et al. Photon counting at telecom wavelengths with commercial InGaAs/InP avalanche photodiodes: current performance. J Mod Opt 2004; 51: 1381-1398.

65 Yoshizawa A, Kaji R, Tsuchida H. Gated-mode single-photon detection at $1550 \mathrm{~nm}$ by discharge pulse counting. Appl Phys Lett 2004; 84: 3606

66 Voss PL, Köprülü KG, Choi S, Dugan S, Kumar P. $14 \mathrm{MHz}$ rate photon counting with room temperature InGaAs/InP avalanche photodiodes. J Mod Opt 2004; 51: 13691379 .

67 Wu G, Zhou C, Chen X, Zeng H. High performance of gated-mode single-photon detector at $1.55 \mu \mathrm{m}$. Opt Commun 2006; 265: 126-131.

68 Liu M, Hu C, Bai X, Guo X, Campbell JC et al. High-performance InGaAs/InP singlephoton avalanche photodiode. IEEE J Sel Top Quantum Electron 2007: 13: 887-894.

69 Dimler SJ, Tozer RC, Rees GJ, David JPR. Capacitive quenching measurement circuit for Geiger-mode avalanche photodiodes. IEEE J Sel Top Quantum Electron 2007; 13 919-925.

70 Tisa S, Guerrieri F, Zappa F. Variable-load quenching circuit for single-photon avalanche diodes. Opt Express 2008; 16: 2232-2244.

71 Dalla Mora A, Tosi A, Zappa F, Cova S, Contini D et al. Fast-gated single-photon avalanche diode for wide dynamic range near infrared spectroscopy. IEEE J Sel Top Quantum Electron 2010; 16: 1023-1030.

72 Cho SK, Kang SK. Weak avalanche discrimination for gated-mode single-photon avalanche photodiodes. Opt Express 2011; 19: 18510-18515.

73 Tosi A, Frera AD, Shehata AB, Scarcella C. Fully programmable single-photon detection module for InGaAs/InP single-photon avalanche diodes with clean and sub-nanosecond gating transitions. Rev Sci Instrum 2012; 83: 013104.

74 Tosi A, Acerbi F, Anti M, Zappa F. InGaAs/InP single-photon avalanche diode with reduced afterpulsing and sharp timing response with 30 ps tail. IEEE J Quantum Electron 2012; 48: 1227-1232.

75 Lu Z, Sun W, Campbell JC, Jiang X, Itzler MA. Pulsed gating with balanced InGaAs/InP single photon avalanche diodes. IEEE J Quantum Electron 2013; 46: 485-490.

76 Namekata N, Sasamori S, Inoue S. $800 \mathrm{MHz}$ Single-photon detection at 1550-nm using an InGaAs/InP avalanche photodiode operated with a sine wave gating. Opt Express 2006; 14: 10043-10049.

77 Zhang J, Thew R, Barreiro C, Zbinden H. Practical fast gate rate InGaAs/InP singlephoton avalanche photodiodes. Appl Phys Lett 2009; 95: 091103.

78 Yuan ZL, Kardynal BE, Sharpe AW, Shields AJ. High speed single photon detection in the near infrared. Appl Phys Lett 2007; 91: 041114

79 Namekata N, Adachi S, Inoue S. $1.5 \mathrm{GHz}$ single-photon detection at telecommunication wavelengths using sinusoidally gated InGaAs/InP avalanche photodiode. Opt Express 2009; 17: 6275-6282.

80 Dixon AR, Dynes JF, Yuan ZL, Sharpe AW, Bennett AJ et al. Ultrashort dead time of photon-counting InGaAs avalanche photodiodes. Appl Phys Lett 2009; 94: 231113.

81 Xu L, Wu E, Gu X, Jian Y, Wu G et al. High-speed InGaAs/InP-based single-photon detector with high efficiency. Appl Phys Lett 2009; 94: 161106.

82 Zhang J, Eraerds P, Walenta N, Barreiro C, Thew R et al. $2.23 \mathrm{GHz}$ gating InGaAs/InP single-photon avalanche diode for quantum key distribution. Proc SPIE 2010; 7681 $76810 Z$

83 Yuan ZL, Sharpe AW, Dynes JF, Dixon AR, Shields AJ. Multi-gigahertz operation of photon counting InGaAs avalanche photodiodes. Appl Phys Lett 2010; 96: 071101.

84 Chen X, Wu E, Wu G, Zeng H. Low-noise high-speed InGaAs/InP-based single-photon detector. Opt Express 2010; 18: 7010-7018.

85 Ren M, Gu X, Liang Y, Kong W, Wu E et al. Laser ranging at $1550 \mathrm{~nm}$ with 1-GHz sinewave gated InGaAs/InP APD single-photon detector. Opt Express 2011; 19: 13497 13502.

86 Nambu Y, Takahashi S, Yoshino K, Tanaka A, Fujiwara M et al. Efficient and low-noise single-photon avalanche photodiode for $1.244-\mathrm{GHz}$ clocked quantum key distribution. Opt Express 2011; 19: 20531-20541.

87 Liang Y, Wu E, Chen X, Ren M, Jian Y et al. Low-timing-jitter single-photon detection using 1-GHz sinusoidally gated InGaAs/InP avalanche photodiode. IEEE Photon Technol Lett 2011; 23: 887-889.

88 Liang XL, Liu JH, Wang Q, Du DB, Ma J et al. Fully integrated InGaAs/InP singlephoton detector module with gigahertz sine wave gating. Rev Sci Instrum 2012; 83: 083111. 
89 Walenta N, Lunghi T, Guinnard O, Houlmann R, Zbinden $\mathrm{H}$ et al. Sine gating detector with simple filtering for low-noise infra-red single photon detection at room temperature. J Appl Phys 2012; 112: 063106.

90 Campbell JC, Sun W, Lu Z, Itzler MA, Jiang X. Common-mode cancellation in sinusoidal gating with balanced InGaAs/InP single photon avalanche diodes. IEEE J Quantum Eletron 2012; 48: 1505-1511.

91 Wu QL, Namekata N, Inoue S. Sinusoidally gated InGaAs avalanche photodiode with direct hold-off function for efficient and low-noise single-photon detection. Appl Phys Express 2013; 6: 062202

92 Lu Z, Sun W, Zhou Q, Campbell J, Jiang X et al. Improved sinusoidal gating with balanced InGaAs/InP Single Photon Avalanche Diodes. Opt Express 2013; 21 $16716-16721$

93 Zhang Y, Zhang X, Wang S. Gaussian pulse gated InGaAs/InP avalanche photodiode for single photon detection. Opt Lett 2013; 38: 606-608.

94 Restelli A, Bienfang JC, Migdall AL. Single-photon detection efficiency up to $50 \%$ at $1310 \mathrm{~nm}$ with an InGaAs/InP avalanche diode gated at $1.25 \mathrm{GHz}$. App/ Phys Lett 2013; 102: 141104.

95 Wu G, Jian Y, Wu E, Zeng H. Photon-number-resolving detection based on InGaAs/InP avalanche photodiode in the sub-saturated mode. Opt Express 2009; 17: 18782 18787

96 Kardynal BE, Yuan ZL, Shields AJ. An avalanche-photodiode-based photon-numberresolving detector. Nat Photonics 2008; 2: 425-428.

97 Thomas O, Yuan ZL, Dynes JF, Sharpe AW, Shields AJ. Efficient photon number detection with silicon avalanche photodiodes. Appl Phys Lett 2010; 97: 031102.

98 Suzuki S, Namekata N, Tsujino K, Inoue S. Highly enhanced avalanche probability using sinusoidally-gated silicon avalanche photodiode. App/ Phys Lett 2014; 104 041105 .

99 Warburton RE, Itzler MA, Buller GS. Free-running, room temperature operation of an InGaAs/InP single-photon avalanche diode. Appl Phys Lett 2009; 94: 071116.

100 Liu M, Hu C, Campbell JC, Pan Z, Tashima MM. Reduce afterpulsing of single photon avalanche diodes using passive quenching with active reset. IEEE J Quantum Electron 2008; 44: 430-434.

$101 \mathrm{Hu}$ C, Liu M, Zheng X, Campbell JC. Dynamic range of passive quenching active rese circuit for single photon avalanche diodes. IEEE J Quantum Electron 2010; 46: 3539.

$102 \mathrm{Hu}$ C, Zheng X, Campbell J, Onat B, Jiang X et al. Characterization of an InGaAs/InP based single-photon avalanche diode with gated-passive quenching with active reset circuit. J Mod Opt 2011; 58: 201-209.

103 Thew RT, Stucki D, Gautier JD, Zbinden H, Rochas A. Free-running InGaAs/InP avalanche photodiode with active quenching for single photon counting at telecom wavelengths. Appl Phys Lett 2007; 91: 201114.

104 Eraerds P, Legré M, Zhang J, Zbinden H, Gisin N. Photon counting OTDR: advantages and limitations. J Lightwave Technol 2010; 28: 952-964.

105 Jiang X, Itzler MA, Nyman B, Slomkowski K. Negative feedback avalanche diodes for near-infrared single photon detection. Proc SPIE 2009; 7320: 732011.

106 Itzler MA, Jiang X, Nyman B, Slomkowski K. InP-based negative feedback avalanche diodes. Proc SPIE 2009; 7222: 72221K.

107 Itzler MA, Jiang X, Onat BM, Slomkowski K. Progress in self-quenching InP-based single photon detectors. Proc SPIE 2010; 7608: 760829.

108 Hayat MM, Itzler MA, Ramirez DA, Rees GJ. Model for passive quenching of SPADs. Proc SPIE 2010; 7608: 76082B.

109 Hayat MM, Ramirez DA, Rees GJ, Itzler MA. Modeling negative feedback in singlephoton avalanche diodes. Proc SPIE 2010; 7681: 76810W

110 Jiang X, Itzler MA, O'Donnell K, Entwistle M, Slomkowski K. InGaAs/InP negative feedback avalanche diodes (NFADs). Proc SPIE 2011; 8033: 80330K.

111 Lunghi T, Barreiro C, Guinnard O, Houlmann R, Jiang X et al. Free running single photon detection based on a negative feedback InGaAs APD. J Mod Opt 2012; 59: 1481-1488.

112 Yan Z, Hamel DR, Heinrichs AK, Jiang X, Itzler MA et al. An ultra low noise telecom wavelength free running single photon detector using negative feedback avalanche diode. Rev Sci Instrum 2012; 83: 073105.

113 Korzh B, Walenta N, Lunghi T, Gisin N, Zbinden H. Free-running InGaAs single photon detector with 1 dark count per second at 10\% efficiency. App/ Phys Lett 2014; 104 081108.

114 Ribordy G, Gautier JD, Gisin N, Gautier O, Zbinden H. Automated "plug \& play" quantum key distribution. Electron Lett 1998; 34: 2116-2117.

115 Bourennane M, Gibson F, Karlsson A, Hening A, Jonsson P et al. Experiments on long wavelength $(1550 \mathrm{~nm})$ plug and play quantum cryptography systems. Opt Express 1999; 4: 383-387.

116 Hughes RJ, Morgan GL, Peterson CG. Quantum key distribution over a 48 km optical fibre network. J Mod Opt 2000; 47: 533-547.

117 Stucki D, Gisin N, Guinnard O, Ribordy G, Zbinden H. Quantum key distribution over $67 \mathrm{~km}$ with a plug\&play system. New J Phys 2002; 4: 41.

118 Kosaka H, Tomita A, Nambu Y, Kimura T, Nakamura K. Single-photon interference experiment over $100 \mathrm{~km}$ for quantum cryptography system using balanced gatedmode photon detector. Electron Lett 2003; 39: 1199-1201.
119 Gobby C, Yuan ZL, Shields AJ. Quantum key distribution over $122 \mathrm{~km}$ of standard telecom fiber. Appl Phys Lett 2004; 84: 3762.

120 Peng CZ, Zhang J, Yang D, Gao WB, Ma HX et al. Experimental long-distance decoystate quantum key distribution based on polarization encoding. Phys Rev Lett 2007; 98: 010505.

121 Namekata N, Fujii G, Inoue S, Honjo T, Takesue H. Differential phase shift quantum key distribution using single-photon detectors based on a sinusoidally gated InGaAs/ InP avalanche photodiode. Appl Phys Lett 2007; 91: 011112.

122 Namekata N, Takesue H, Honjo T, Tokura Y, Inoue S. High-rate quantum key distribution over $100 \mathrm{~km}$ using ultra-low-noise, 2-GHz sinusoidally gated InGaAs/ InP avalanche photodiodes. Opt Express 2011; 19: 10632-10639.

123 Yuan ZL, Dixon AR, Dynes JF, Sharpe AW, Shields AJ. Gigahertz quantum key distribution with InGaAs avalanche photodiodes. App/ Phys Lett 2008; 92: 201104.

124 Yuan ZL, Dixon AR, Dynes JF, Sharpe AW, Shields AJ. Practical gigahertz quantum key distribution based on avalanche photodiodes. New J Phys 2009; 11: 045019.

125 Dixon AR, Yuan ZL, Dynes JF, Sharpe AW, Shields AJ. Continuous operation of high bit rate quantum key distribution. Appl Phys Lett 2010; 96: 161102.

126 Patel KA, Dynes JF, Lucamarini M, Choi I, Sharpe AW et al. Quantum key distribution for $10 \mathrm{~Gb} / \mathrm{s}$ dense wavelength division multiplexing networks. Appl Phys Lett 2014; 104: 051123.

127 Comandar LC, Fröhlich B, Lucamarini M, Patel KA, Sharpe AW et al. Room temperature single-photon detectors for high bit rate quantum key distribution. App/ Phys Lett 2014; 104: 021101.

128 Korzh B, Lim CCW, Houlmann R, Gisin N, Li MJ et al. Provably secure and practical quantum key distribution over $307 \mathrm{~km}$ of optical fibre. Nat Photonics 2015; 9: 163-168.

129 Marcikic I, Riedmatten H, Tittel W, Zbinden H, Legré M et al. Distribution of time-bin entangled qubits over 50 km of optical fiber. Phys Rev Lett 2004; 93: 180502.

130 Takesue H. Long-distance distribution of time-bin entanglement generated in a cooled fiber. Opt Express 2006; 14: 3453-3460.

131 Hübel H, Vanner MR, Lederer T, Blauensteiner B, Lorünser T et al. High-fidelity transmission of polarization encoded qubits from an entangled source over $100 \mathrm{~km}$ of fiber. Opt Express 2007; 15: 7853-7862.

132 Dynes JF, Takesue H, Yuan ZL, Sharpe AW, Harada K et al. Efficient entanglement distribution over 200 kilometers. Opt Express 2009; 17: 11440-11449.

133 Marcikic I, Riedmatten H, Tittel W, Zbinden H, Gisin N. Long distance teleportation of qubits at telecommunication wavelengths. Nature 2003; 421: 509-513.

134 Riedmatten $\mathrm{H}$, Marcikic I, Tittel W, Zbinden H, Collins D et al. Long distance quantum teleportation in a quantum relay configuration. Phys Rev Lett 2004; 92: 047904.

135 Landry O, Houwelingen JAW, Beveratos A, Zbinden H, Gisin N. Quantum teleportation over the Swisscom telecommunication network. J Opt Soc Am B2007; 24: 398-403.

136 Bogdanski J, Rafiei N, Bourennane M. Experimental quantum secret sharing using telecommunication fiber. Phys Rev A 2008; 78: 062307.

$137 \mathrm{Ma} \mathrm{HQ}$, Wei KJ, Yang JH. Experimental single qubit quantum secret sharing in a fiber network configuration. Opt Lett 2013; 38: 4494-4497.

138 Wei KJ, Ma HQ, Yang JH. Experimental circular quantum secret sharing over telecom fiber network. Opt Express 2013; 21: 16663-16669.

139 Ren M, Wu G, Wu E, Zeng HP. Experimental demonstration of counterfactual quantum key distribution. Laser Phys 2011; 21: 755-760.

140 Liu Y, Ju L, Liang XL, Tang SB, Shentu GL et al. Experimental demonstration of counterfactual quantum communication. Phys Rev Lett 2012; 109: 030501.

141 Zhao K, You S, Cheng J, Lo YH. Self-quenching and self-recovering InGaAs/InAIAs single photon avalanche detector. Appl Phys Lett 2008; 93: 153504

142 Cheng J, You S, Rahman S, Lo YH. Self-quenching InGaAs/InP single photon avalanche detector utilizing zinc diffusion rings. Opt Express 2011; 19: 1514915154.

143 Zappa F, Ghioni M, Cova S, Samori C, Giudice AC. An integrated active-quenching circuit for single-photon avalanche diodes. IEEE T Instrum Meas 2000; 49: 11671175.

144 Zappa F, Lotito A, Giudice AC, Cova S, Ghioni M. Monolithic active-quenching and active-reset circuit for single-photon avalanche detectors. IEEE J Solid-State Circuits 2003; 38: 1298-1301.

145 Zappa F, Tisa S, Gulinatti A, Gallivanoni A, Cova S. Complete single-photon counting and timing module in a microchip. Opt Lett 2005; 30: 1327-1329.

146 Deng S, Morrison AP. Active quench and reset integrated circuit with novel hold-off time control logic for Geiger-mode avalanche photodiodes. Opt Lett 2012; 37: 38763878.

(c) (i) (2) (2) This work is licensed under a Creative Commons Attribution-

cc. ${ }_{\mathrm{BY}} \mathrm{NC}$ SA NonCommercial-ShareAlike 3.0 Unported License. The images or other third party material in this article are included in the article's Creative Commons license, unless indicated otherwise in the credit line; if the material is not included under the Creative Commons license, users will need to obtain permission from the license holder to reproduce the material. To view a copy of this license, visit http://creativecommons.org/licenses/by-nc-sa/3.0/ 